\title{
Coherent Transport in Periodically Driven Mesoscopic Conductors: From Scattering Amplitudes to Quantum Thermodynamics
}

https://doi.org/10.1515/zna-2020-0056

Received February 22, 2020; accepted March 8, 2020

\begin{abstract}
Scattering theory is a standard tool for the description of transport phenomena in mesoscopic systems. Here, we provide a detailed derivation of this method for nano-scale conductors that are driven by oscillating electric or magnetic fields. Our approach is based on an extension of the conventional Lippmann-Schwinger formalism to systems with a periodically time-dependent Hamiltonian. As a key result, we obtain a systematic perturbation scheme for the Floquet scattering amplitudes that describes the transition of a transport carrier through a periodically driven sample. Within a general multiterminal setup, we derive microscopic expressions for the mean values and time-integrated correlation functions, or zero-frequency noise, of matter and energy currents, thus recovering the results of earlier studies in a unifying framework. We show that this framework is inherently consistent with the first and the second law of thermodynamics and prove that the mean rate of entropy production vanishes only if all currents in the system are zero. As an application, we derive a generalized Green-Kubo relation, which makes it possible to express the response of any mean currents to small variations of temperature and chemical potential gradients in terms of time integrated correlation functions between properly chosen currents. Finally, we discuss potential topics for future studies and further reaching applications of the Floquet scattering approach to quantum transport in stochastic and quantum thermodynamics.
\end{abstract}

Keywords: Current Fluctuations in Non-Equilibrium Systems; Floquet Theory; Quantum Thermodynamics; Quantum Transport; Scattering Theory.

\footnotetext{
*Corresponding author: Kay Brandner, Department of Physics, Keio University, 3-14-1 Hiyoshi, Kohoku-ku, Yokohama 223-8522, Japan; School of Physics and Astronomy, University of Nottingham, Nottingham NG7 2RD, UK; and Centre for the Mathematics and Theoretical Physics of Quantum Non-equilibrium Systems, University of Nottingham, Nottingham NG7 2RD, UK, E-mail: kay.brandner@nottingham.ac.uk.https://orcid.org/00000002-4425-8252
}

\section{Introduction}

At room temperature, transport in macroscopic systems is a stochastic process, where carriers undergo ceaseless collisions that randomly change their velocity and direction of motion. This irregular behavior is the microscopic origin of both the finite resistance of a normal conductor and the fluctuations of induced currents. The fundamental relationship between these two phenomena is described by the fluctuation-dissipation theorem, a cornerstone result of statistical mechanics, which goes back to the pioneering works of Einstein, Nyquist, and Onsager and was later derived in a unified manner by Callen and Welton. Green and Kubo further expanded this approach and showed that, close to equilibrium, linear transport coefficients, which describe the response of a system to a small external field or thermal perturbation, can be expressed in terms of time integrated correlation functions of the corresponding currents, i.e. the zero-frequency noise [1-3]. This universal structure can be recovered even for systems in non-equilibrium steady states by introducing more general correlation functions that involve a current and a suitably chosen conjugate variable [4].

Reducing the temperature of a conductor increases the average distance that carriers can travel between two consecutive collisions. Coherent transport sets in when this mean free path becomes comparable to the dimensions of the sample. In this regime, which is realized in mesoscopic systems at millikelvin temperatures, the transfer of carriers becomes a reversible process governed by Schrödinger's equation. As a result, the properties of mesoscopic conductors are dominated by quantum effects such as conductance quantization or coherent resistance oscillations, which can no longer be understood in terms of classical stochastic trajectories [5-7].

Scattering theory provides a quantum mechanical description of open systems that are subject to a constant in- and outflow of particles. Therefore, it is a well-suited tool to explore the principles of coherent transport. This approach was first proposed by Landauer and has since then evolved into a powerful theoretical framework, which has been extensively tested in experiments and shaped our modern understanding of transport phenomena in 
small-scale conductors. At the core of this framework lies the Landauer-Büttiker formula. It connects the scattering amplitudes of a mesoscopic sample, which describe the elastic deflection of incoming carriers, with the matter and energy currents that emerge in the system under external biases. Hence, it provides a direct link between microscopic and macroscopic quantities [5-10].

As a key application, the scattering approach to quantum transport enables systematic investigations of the elementary principles that govern the thermodynamics of mesoscopic conductors and the performance of autonomous nano-machines such as thermoelectric heat engines or refrigerators [11-14]. Cyclic machines like charge pumps or quantum motors, however, require the input or extraction of mechanical work; therefore, they must be driven by time-dependent electric or magnetic fields, which alter the energy of carriers inside the sample. Floquet theory provides an elegant way to take this effect into account by introducing a new type of scattering amplitudes that describe inelastic transitions, where carriers exchange photons with the external fields. This Floquet scattering approach yields a generalized LandauerBüttiker formula for periodically driven systems [15-19]. Among other applications, this result enables quantitative models for cyclic nano-machines, which can be used to develop practical devices or to explore fundamental performance limits, two central topics in the field of quantum thermodynamics [20, 21].

The Floquet scattering approach also leads to explicit microscopic expressions for the time integrated correlation functions of matter and energy currents in periodically driven quantum conductors [22-24]. It thus provides a powerful tool to investigate the complex interplay between dissipation, thermal, and quantum fluctuations in mesocopic systems. This topic includes the search for generalizations of the well-established Green-Kubo relations as well as the quest for quantum extensions of the recently discovered thermodynamic uncertainty relations [25].

\section{Objective and Outline}

Our aim is to provide a thorough and general derivation of the Floquet scattering approach to coherent transport in mesoscopic conductors. This article is supposed to serve as both a step-by-step introduction for new users of the formalism and a compact reference text for experts in the field. We do not attempt to give a complete overview of the existing literature. Instead, our objective is to complement earlier works by focusing on the development of an algebraic scattering theory for periodically driven mesoscopic conductors and applications in stochastic and quantum thermodynamics.

We proceed as follows. In Section 3, we set the stage for our analysis by introducing the multi-terminal model as a general basis for the discussion of coherent transport. This section is followed by a brief recap of the algebraic scattering theory for autonomous systems in Section 4, which is based on common textbooks [6, 7, 26, 27]. We then show how the Floquet theorem makes it possible to extend this framework to periodically driven systems in Section 5. Following the approach of earlier studies, we construct an extended Hilbert space, which was originally proposed for closed systems [28], to derive a generalized LippmannSchwinger equation for Floquet scattering states [29-31]. This result naturally leads to a systematic perturbation scheme for the crucial Floquet scattering amplitudes and to explicit expressions for the corresponding scattering wave functions, which enable a transparent physical interpretation of the formalism.

In Section 6 we switch from the single-particle picture that had been used in the foregoing sections to a many-particle description. To this end we first show how the operators $\mathrm{J}_{\alpha, t}^{\rho}$ and $\mathrm{J}_{\alpha, t}^{\varepsilon}$, which represent the matter and energy currents in a multi-terminal conductor at the time $t$, can be connected to the previously discussed Floquet scattering states. We then derive microscopic expressions for the mean currents and the time integrated current correlation functions, or noise power, which are given by

$$
\begin{aligned}
J_{\alpha}^{X} & \equiv \lim _{t \rightarrow \infty} \frac{1}{t} \int_{0}^{t} \mathrm{~d} t^{\prime}\left\langle\mathrm{J}_{\alpha, t}^{X}\right\rangle \quad \text { and } \\
P_{\alpha \beta}^{x y} & \equiv \lim _{t \rightarrow \infty} \frac{1}{t} \int_{0}^{t} \mathrm{~d} t^{\prime} \int_{0}^{t} \mathrm{~d} t^{\prime \prime}\left\langle\left(\mathrm{J}_{\alpha, t^{\prime}}^{x}-J_{\alpha}^{x}\right)\left(\mathrm{J}_{\beta, t^{\prime \prime}}^{y}-J_{\beta}^{y}\right)\right\rangle,
\end{aligned}
$$

where angular brackets denote the average over all possible quantum sates of the system. We thereby recover the results of earlier studies [15, 22, 24].

Moving on, in Section 7 we show how the Floquet scattering approach can be furnished with a thermodynamic structure. To this end, we formulate the first and the second law and show that the scattering formalism is inherently consistent with these constraints. As an application of this theory, we derive a generalization of the Green-Kubo relations for periodically driven systems far from equilibrium. Finally, we discuss open problems and potential starting points for future studies in Section (8). 


\section{The Multi-Terminal Model}

The multi-terminal model provides a universal platform for the description of coherent transport in mesoscopic systems. The key idea is thereby to divide the conductor into a scattering region, where carriers are affected by the potential landscape of the sample and periodic driving fields, and a set of $N$ ideal leads, which can be traversed freely (Fig. 1). For the sake of simplicity, we assume throughout this article that the leads are effectively onedimensional. ${ }^{1}$

Each lead is connected to a thermochemical reservoir with a fully transparent interface, which injects a continuous beam of thermalized, non-interacting carriers into the system. Inside the conductor, these carriers follow a deterministic time evolution governed by Schrödinger's equation until they are absorbed again into one of the reservoirs. Hence, all irreversible processes are relegated to the reservoirs, while the transfer of carriers between them is coherent. Once the system has reached a steady state, each lead $\alpha$ is traversed by a periodically modulated beam of incoming and outgoing carries, which gives rise to a matter and an energy current. The corresponding mean values and fluctuations are given by the formulas (1). As we will see in the following sections, these quantities are completely determined by the scattering amplitudes of the driven sample and the energy distribution of the carriers injected by the reservoirs.

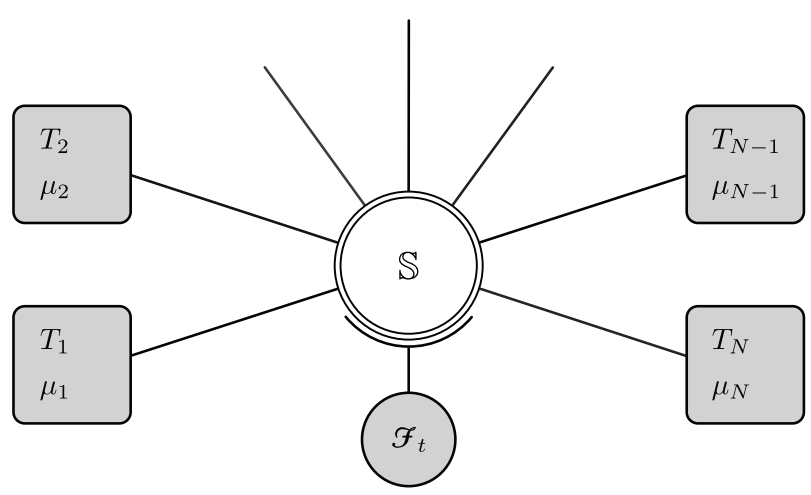

Figure 1: Sketch of the multi-terminal model for a generic mesoscopic conductor. A central scattering region, or sample, $\mathbb{S}$ is connected via ideal, one-dimensional leads to $N$ heat and particle reservoirs with temperatures $T_{1}, \ldots, T_{N}$ and chemical potentials $\mu_{1}, \ldots, \mu_{N}$. The external driving fields $\mathcal{F}_{t}$ periodically change the potential inside the scattering region.

1 Specifically, we assume that the waveguides are so narrow that only the lowest transverse modes contribute to the transport process in the relevant range of energies, for details see [7].

\section{Standard Scattering Theory}

\subsection{Scattering States}

Without external driving, the carrier dynamics in a multiterminal system is governed by the Hamiltonian

$$
H=P^{2} / 2 M+U \text {. }
$$

Here $P$ and $M$ are the carrier momentum and mass and $U$ accounts for the potential landscape of the scattering region as well as the coupling to external magnetic fields. The scattering of individual carriers with fixed energy $E>0$ is described by solutions of the time-dependent Schrödinger equation that have the form

$$
\left|\psi_{E, t}^{\alpha \pm}\right\rangle=\exp [-i E t / \hbar]\left|\varphi_{E}^{\alpha \pm}\right\rangle .
$$

The outgoing and incoming states, $\left|\varphi_{E}^{\alpha+}\right\rangle$ and $\left|\varphi_{E}^{\alpha-}\right\rangle$, thereby represent carriers that enter and escape the system through the terminal $\alpha$, respectively. These scattering states satisfy the stationary Schrödinger equation

$$
H\left|\varphi_{E}^{\alpha \pm}\right\rangle=E\left|\varphi_{E}^{\alpha \pm}\right\rangle
$$

and the boundary conditions

$$
\left\langle r_{\beta} \mid \varphi_{E}^{\alpha \pm}\right\rangle \equiv \varphi_{E}^{\alpha \pm}\left[r_{\beta}\right]=\delta_{\alpha \beta} w_{E}^{\mp}\left[r_{\beta}\right]+S_{E}^{\alpha \beta \pm} w_{E}^{ \pm}\left[r_{\beta}\right] .
$$

Here, the plane waves

$$
w_{E}^{ \pm}[r] \equiv \xi_{E} \exp \left[ \pm i k_{E} r\right] \quad \text { with } \quad k_{E} \equiv \sqrt{2 M E / \hbar^{2}}
$$

describe the free propagation of carriers inside the leads and the scattering amplitudes, $S_{E}^{\alpha \beta+}$ and $S_{E}^{\alpha \beta-}=\bar{S}_{E}^{\beta \alpha+}$, account for transition between the terminals $\beta$ and $\alpha^{2}$. The coordinate $r_{\beta} \geq 0$ parameterizes the lead $\beta$ in radial direction and the factor

$$
\xi_{E} \equiv \sqrt{\left(\mathrm{d} k_{E} / \mathrm{d} E\right) / 2 \pi}=\sqrt{M / 2 \pi k_{E} \hbar^{2}}
$$

has been introduced for normalization [32].

The outgoing and incoming states as defined by the conditions (4) and (5) obey the orthogonality relations

$$
\left\langle\varphi_{E^{\prime}}^{\beta \pm} \mid \varphi_{E}^{\alpha \pm}\right\rangle=\delta_{\alpha \beta} \delta_{E-E^{\prime}}
$$

and form two complete bases of the single-particle Hilbert space $\mathcal{H}$; for simplicity, we assume throughout this article that no bound states exist inside the scattering region ${ }^{3}$.

2 Throughout this article, bars indicate complex conjugation. 3 Note that the bound states generally affect the properties of mesoscopic conductors although they do not contribute directly to the transport process [33]. 
The scattering states are not normalizable and carry a finite probability current. Therefore, they cannot be interpreted in the same manner as bound states, whose wave function corresponds to the probability amplitude for finding a particle at a given position. Instead, we may regard the scattering states as a quantum mechanical description of a homogeneous sequence of carriers that emerge from a distant source and travel through the system one by one before being absorbed by a distant sink [26]. This interpretation does not imply that the states $\left|\varphi_{E}^{\alpha \pm}\right\rangle$ represent more than one particle; it rather entails that they describe a large number of identical and independent scattering experiments [34]. In this picture, the square modulus of the scattering amplitude $S_{E}^{\alpha \beta+}$ is the probability for a carrier with energy $E$ that is injected into the terminal $\alpha$ to leave the system through the terminal $\beta$. Analogously, the square modulus of $S_{E}^{\alpha \beta-}$ is the probability for a carrier with energy $E$ that escapes through the terminal $\alpha$ to originate form the terminal $\beta$.

\subsection{Scattering Amplitudes}

To ensure the conservation of probability currents, the scattering amplitudes have to obey the unitarity condition [32]

$$
\sum_{\gamma} S_{E}^{\alpha \gamma \pm} S_{E}^{\gamma \beta \mp}=\delta_{\alpha \beta} .
$$

Furthermore, they provide a link between outgoing and incoming states by means of the relation

$$
\left|\varphi_{E}^{\alpha \pm}\right\rangle=\sum_{\beta} S_{E}^{\alpha \beta \pm}\left|\varphi_{E}^{\beta \mp}\right\rangle,
$$

which can be easily verified in position representation using the boundary conditions (5) and (9).

Upon applying the orthogonality relation (8), (10) implies an algebraic expression for the scattering amplitudes in terms of the scattering states given by

$$
\left\langle\varphi_{E^{\prime}}^{\beta \mp} \mid \varphi_{E}^{\alpha \pm}\right\rangle=S_{E}^{\alpha \beta \pm} \delta_{E-E^{\prime}}
$$

This result makes it possible to establish a universal symmetry, which follows from the observation that outgoing and incoming states are connected by time reversal, i.e.

$$
\left|\varphi_{E}^{\alpha \pm}\right\rangle=\Theta\left|\tilde{\varphi}_{E}^{\alpha \mp}\right\rangle,
$$

where $\Theta$ denotes the anti-unitary time-reversal operator [35] and tildes indicate the reversal of external magnetic fields, see Figure 2. Consequently, we have

$$
\left\langle\varphi_{E^{\prime}}^{\beta \mp} \mid \varphi_{E}^{\alpha \pm}\right\rangle=\left\langle\Theta \tilde{\varphi}_{E^{\prime}}^{\beta \pm} \mid \Theta \tilde{\varphi}_{E}^{\alpha \mp}\right\rangle=\left\langle\tilde{\varphi}_{E}^{\alpha \mp} \mid \tilde{\varphi}_{E^{\prime}}^{\beta \pm}\right\rangle=\tilde{S}_{E}^{\beta \alpha \pm} \delta_{E-E^{\prime}}
$$
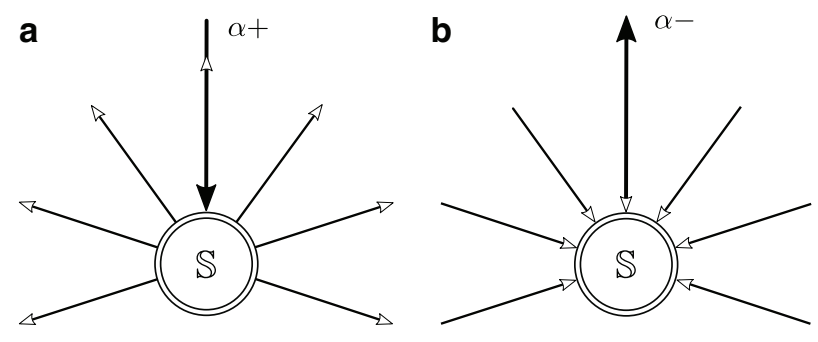

Figure 2: Schematic representation of incoming and outgoing scattering states. (a) The incoming state $\left|\varphi_{E}^{\alpha+}\right\rangle$ consists of a plane wave with energy $E$ that approaches the sample in the terminal $\alpha$ and decomposes into $N$ escaping waves with the same energy but smaller amplitude. (b) The outgoing state $\left|\varphi_{E}^{\alpha-}\right\rangle$ describes the time-reversed situation, where $N$ approaching waves with the same energy $E$ combine into a single one that escapes through the terminal $\alpha$, cf. (5).

and therefore, given the (11),

$$
S_{E}^{\alpha \beta \pm}=\tilde{S}_{E}^{\beta \alpha \pm} .
$$

Hence, for systems without magnetic fields, the scattering amplitudes for forward and backward transitions between any two terminals $\alpha$ and $\beta$ are identical.

\subsection{Lippmann-Schwinger Theory I: Autonomous Systems}

The scattering states and amplitudes can, in principle, be determined by rewriting the stationary Schrödiner equation (4) in position representation, calculating the wave function inside the scattering region and matching it with the boundary conditions (5). This procedure, however, becomes impractical when the scattering wave functions cannot be found exactly and perturbation methods must be applied. It is then more convenient to follow an algebraic approach, which we develop next.

We first divide the Hamiltonian (2) into a free part $H_{0}$ and a perturbation $V$ acting only on the scattering region,

$$
H=H_{0}+V \text {, }
$$

where we assume that the scattering states for $H_{0}$ can be determined exactly. Next, we combine the stationary Schrödinger equations for the free and the perturbed scattering states,

$$
H_{0}\left|\varphi_{0 E}^{\alpha \pm}\right\rangle=E\left|\varphi_{0 E}^{\alpha \pm}\right\rangle \quad \text { and } \quad H\left|\varphi_{E}^{\alpha \pm}\right\rangle=E\left|\varphi_{E}^{\alpha \pm}\right\rangle
$$

into a single inhomogeneous linear equation,

$$
\left[E-H_{0}\right]\left[\left|\varphi_{E}^{\alpha \pm}\right\rangle-\left|\varphi_{0 E}^{\alpha \pm}\right\rangle\right]=V\left|\varphi_{E}^{\alpha \pm}\right\rangle .
$$


This equation can be formally solved for the vector $\left|\varphi_{E}^{\alpha \pm}\right\rangle-\left|\varphi_{0 E}^{\alpha \pm}\right\rangle$ after making the operator $E-H_{0}$ invertible by adding a small imaginary shift. Following these steps, we arrive at the Lippmann-Schwinger equation

$$
\left|\varphi_{E}^{\alpha \pm}\right\rangle=\left|\varphi_{0 E}^{\alpha \pm}\right\rangle+\left[E-H_{0} \pm i \varepsilon\right]^{-1} V\left|\varphi_{E}^{\alpha \pm}\right\rangle,
$$

where $\varepsilon>0$ and the limit $\varepsilon \rightarrow 0$ must be taken after physical observables have been calculated. Note that the sign of the complex shift is important to ensure the correct correspondence between free and perturbed outgoing and incoming states, for details see [26].

By construction, the solutions $\left|\varphi_{E}^{\alpha \pm}\right\rangle$ of (18) also solve the corresponding stationary Schrödinger equation. However, the Lippmann-Schwinger equation contains more information, as it explicitly includes the continuity condition

$$
\lim _{V \rightarrow 0}\left|\varphi_{E}^{\alpha \pm}\right\rangle=\left|\varphi_{0 E}^{\alpha \pm}\right\rangle,
$$

which ensures that the perturbed states $\left|\varphi_{E}^{\alpha \pm}\right\rangle$ obey the boundary conditions (5). That is, for a given set of free states $\left|\varphi_{0 E}^{\alpha \pm}\right\rangle$, the Lippmann-Schwinger equation uniquely determines the outgoing and incoming states for the full Hamiltonian $H$, while the solutions of the stationary Schrödinger equation are unique only up to linear combinations of scattering states with the same energy [26].

The Lippmann-Schwinger equation (18) can be formally solved by iteration. This procedure yields

$$
\begin{aligned}
\left|\varphi_{E}^{\alpha \pm}\right\rangle & =\sum_{l=0}^{\infty}\left[\left[E-H_{0} \pm i \varepsilon\right]^{-1} V\right]^{l}\left|\varphi_{0 E}^{\alpha \pm}\right\rangle \\
& =\left[1-\left[E-H_{0} \pm i \varepsilon\right]^{-1} V\right]^{-1}\left|\varphi_{0 E}^{\alpha \pm}\right\rangle \\
& =\left|\varphi_{0 E}^{\alpha \pm}\right\rangle+[E-H \pm i \varepsilon]^{-1} V\left|\varphi_{0 E}^{\alpha \pm}\right\rangle
\end{aligned}
$$

where the last line follows by noting that

$$
\begin{aligned}
& {\left[1-\left[E-H_{0} \pm i \varepsilon\right]^{-1} V\right]^{-1}} \\
& =\left[\left[E-H_{0} \pm i \varepsilon\right]^{-1}[E-H \pm i \varepsilon]\right]^{-1} \\
& =[E-H \pm i \varepsilon]^{-1}\left[E-H_{0} \pm i \varepsilon\right] \\
& =1+[E-H+ \pm i \varepsilon]^{-1} V .
\end{aligned}
$$

The expression (20) provides a systematic expansion of the scattering states $\left|\varphi_{E}^{\alpha \pm}\right\rangle$ in terms of the perturbation $V$. Moreover, it implies that the solutions of the LippmannSchwinger equation obey the same orthogonality relation as the free states, as

$$
\begin{aligned}
\left\langle\varphi_{E^{\prime}}^{\beta \pm} \mid \varphi_{E}^{\alpha \pm}\right\rangle & =\left\langle\varphi_{0 E^{\prime}}^{\beta \pm} \mid \varphi_{E}^{\alpha \pm}\right\rangle+\frac{\left\langle\varphi_{0 E^{\prime}}^{\beta \pm}|V| \varphi_{E}^{\alpha \pm}\right\rangle}{E^{\prime}-E \mp i \varepsilon} \\
& =\left\langle\varphi_{0 E^{\prime}}^{\beta \pm} \mid \varphi_{0 E}^{\alpha \pm}\right\rangle=\delta_{\alpha \beta} \delta_{E-E^{\prime}}
\end{aligned}
$$

Here, we have first inserted (20) for $\left|\varphi_{E^{\prime}}^{\beta \pm}\right\rangle$ and then (18) for $\left|\varphi_{E}^{\alpha \pm}\right\rangle$ in the first summand. Along the same lines, we find

$$
\begin{aligned}
\left\langle\varphi_{E^{\prime}}^{\beta \mp} \mid \varphi_{E}^{\alpha \pm}\right\rangle & =\left\langle\varphi_{O E^{\prime}}^{\beta \mp} \mid \varphi_{E}^{\alpha \pm}\right\rangle+\frac{\left\langle\varphi_{0 E^{\prime}}^{\beta \mp}|V| \varphi_{E}^{\alpha \pm}\right\rangle}{E^{\prime}-E \pm i \varepsilon} \\
& =\left\langle\varphi_{0 E^{\prime}}^{\beta \mp} \mid \varphi_{0 E}^{\alpha \pm}\right\rangle \mp \frac{2 i \varepsilon}{\left(E-E^{\prime}\right)^{2}+\varepsilon^{2}}\left\langle\varphi_{0 E^{\prime}}^{\beta \mp}|V| \varphi_{E}^{\alpha \pm}\right\rangle \\
& =\left(S_{O E}^{\alpha \beta \pm} \mp 2 \pi i\left\langle\varphi_{0 E^{\prime}}^{\beta \mp}|V| \varphi_{E}^{\alpha \pm}\right\rangle\right) \delta_{E-E^{\prime}},
\end{aligned}
$$

where we have used the relation [36]

$$
\lim _{\varepsilon \rightarrow 0} \frac{\varepsilon}{a^{2}+\varepsilon^{2}}=\pi \delta_{a}
$$

which must be understood in the sense of distributions, and $S_{O E}^{\alpha \beta \pm}$ denotes the scattering amplitudes for the free Hamiltonian $H_{0}$. Comparing (23) with (11) yields the formula

$$
S_{E}^{\alpha \beta \pm}=S_{0 E}^{\alpha \beta \pm} \mp 2 \pi i\left\langle\varphi_{0 E}^{\beta \mp}|V| \varphi_{E}^{\alpha \pm}\right\rangle,
$$

which makes it possible to calculate the full scattering amplitudes order by order in $V$ by using the expansion (20) of the scattering states $\left|\varphi_{E}^{\alpha \pm}\right\rangle$. This perturbation scheme is a key result of the Lippmann-Schwinger formalism and will be developed further in the next section.

\section{Floquet Scattering Theory}

\subsection{Floquet Theory}

The carrier dynamics in a driven multi-terminal system is governed by a Hamiltonian with the general form

$$
H_{t}=P^{2} / 2 M+U+V_{t}=H+V_{t},
$$

where the dynamical potential $V_{t}$ accounts for timedependent external fields acting on the scattering region. If the driving is periodic with frequency $\omega \equiv 2 \pi / \tau$, according to the Floquet theorem, the time-dependent Schrödinger equation admits a complete set of solutions that have the structure

$$
\left|\psi_{E, t}^{\alpha}\right\rangle=\exp [-i E t / \hbar]\left|\phi_{E, t}^{\alpha}\right\rangle,
$$

where $\left|\phi_{E, t+\tau}^{\alpha}\right\rangle=\left|\phi_{E, t}^{\alpha}\right\rangle$, the parameter $E$ here plays the role of a continuous quantum number and $\alpha$ stands for any discrete quantum number [28, 37, 38]. The Floquet states $\left|\phi_{E, t}^{\alpha}\right\rangle$ obey the Floquet-Schrödinger equation

$$
\left[H_{t}-i \hbar \partial_{t}\right]\left|\phi_{E, t}^{\alpha}\right\rangle=E\left|\phi_{E, t}^{\alpha}\right\rangle
$$


and form an orthogonal basis of the single-particle Hilbert space $\mathcal{H}$ at every fixed time $t$.

In order to formulate a systematic scattering theory for periodically driven systems, it is convenient to introduce the extended Hilbert space [28]

$$
\hat{\mathcal{H}} \equiv \mathcal{H} \otimes \mathcal{H}_{\tau},
$$

where $\mathcal{H}_{\tau}$ denotes the Hilbert space of $\tau$-periodic functions. In time representation, the elements $|\psi\rangle\rangle$ of $\hat{\mathcal{H}}$ are $\tau$-periodic single-particle state vectors, i.e.

$$
\langle t \mid \psi\rangle\rangle=\left|\psi_{t}\right\rangle \quad \text { with } \quad\left|\psi_{t+\tau}\right\rangle=\left|\psi_{t}\right\rangle \in \mathcal{H} .
$$

The scalar product in $\hat{\mathcal{H}}$ is defined as

$$
\langle\langle\psi \mid \chi\rangle\rangle \equiv \frac{1}{\tau} \int_{0}^{\tau} \mathrm{d} t\left\langle\psi_{t} \mid \chi_{t}\right\rangle .
$$

This framework makes it possible to cast the FloquetSchrödinger equation (28) into the form of a stationary Schrödinger equation given by

$$
\left.\left.\hat{H}\left|\phi_{E}^{m \alpha}\right\rangle\right\rangle=E_{m}\left|\phi_{E}^{m \alpha}\right\rangle\right\rangle \quad \text { with } \quad E_{m} \equiv E+m \hbar \omega,
$$

where $m$ runs over all integers. The Floquet vectors $\left.\left|\phi_{E}^{m \alpha}\right\rangle\right\rangle$ are connected to the Floquet states according to

$$
\left.\left\langle t \mid \phi_{E}^{m \alpha}\right\rangle\right\rangle=u_{t}^{m}\left|\phi_{E, t}^{\alpha}\right\rangle \quad \text { with } \quad u_{t}^{m} \equiv \exp [i m \omega t]
$$

and the effective Hamiltonian $\hat{H}$, which is defined as

$$
\langle t|\hat{H}| \psi\rangle\rangle \equiv\left[H_{t}-i \hbar \partial_{t}\right]\left|\psi_{t}\right\rangle,
$$

is a self-adjoint operator on $\hat{\mathcal{H}}$ with respect to the scalar product (31). The additional Fourier factor in (33), which is accounted for by the mode index $m$, was introduced to ensure that the solutions of (32) are complete in $\hat{\mathcal{H}}$; this property will be required to develop an algebraic scattering theory in the extended Hilbert space. Once the Floquet vectors $\left.\left|\phi_{E}^{m \alpha}\right\rangle\right\rangle$ have been determined, a complete set of Floquet states $\left|\phi_{E, t}^{\alpha}\right\rangle$ that fulfill (28) is obtained by setting the mode index to zero and returning to the time representation.

\subsection{Lippmann-Schwinger Theory II: Driven Systems}

Replacing the stationary Schrödinger equation (4) with (32), we can now extend the Lippmann-Schwinger theory of autonomous systems to systems with periodic driving. The dynamical potential $V_{t}$ thereby plays the role of the perturbation and the free states are replaced by the Floquet vectors

$$
\left.\left\langle t \mid \varphi_{E}^{m \alpha \pm}\right\rangle\right\rangle \equiv u_{t}^{m}\left|\varphi_{E}^{\alpha \pm}\right\rangle,
$$

where $\left|\varphi_{E}^{\alpha \pm}\right\rangle$ are the scattering states for stationary part $H$ of the Hamiltonian (26). The free Floquet scattering vectors $\left.\left|\varphi_{E}^{m \alpha \pm}\right\rangle\right\rangle$ form a complete basis of the extended Hilbert space $\hat{\mathcal{H}}$, for outgoing and incoming orientation, respectively, and fulfill the Floquet-Schrödinger equation

$$
\left.\left.\hat{H}_{0}\left|\varphi_{E}^{m \alpha \pm}\right\rangle\right\rangle=E_{m}\left|\varphi_{E}^{m \alpha \pm}\right\rangle\right\rangle
$$

where the free effective Hamiltonian is defined as

$$
\left.\left\langle t\left|\hat{H}_{0}\right| \psi\right\rangle\right\rangle=\left[H-i \hbar \partial_{t}\right]\left|\psi_{t}\right\rangle .
$$

Furthermore, using (8) and (31), it is straightforward to verify the orthogonality relation

$$
\left\langle\left\langle\varphi_{E^{\prime}}^{n \beta \pm} \mid \varphi_{E}^{m \alpha \pm}\right\rangle\right\rangle=\delta_{m n} \delta_{\alpha \beta} \delta_{E-E^{\prime}} .
$$

Note that the quantum numbers $E$ and $\alpha$ have now been identified with the energy and the terminal of either an incident $(+)$ or an escaping $(-)$ carrier.

The full Floquet scattering vectors $\left.\left|\phi_{E}^{m \alpha \pm}\right\rangle\right\rangle$ are those solutions of the Floquet-Schrödinger equation

$$
\left.\left.\hat{H}\left|\phi_{E}^{m \alpha \pm}\right\rangle\right\rangle=E_{m}\left|\phi_{E}^{m \alpha \pm}\right\rangle\right\rangle
$$

that reduce to the corresponding free vectors $\left.\left|\varphi_{E}^{m \alpha \pm}\right\rangle\right\rangle$ in the stationary limit $V_{t} \rightarrow 0$. They are uniquely determined by the Floquet-Lippmann-Schwinger equation

$$
\left.\left.\left.\left|\phi_{E}^{m \alpha \pm}\right\rangle\right\rangle=\left|\varphi_{E}^{m \alpha \pm}\right\rangle\right\rangle+\left[E_{m}-\hat{H}_{0} \pm i \varepsilon\right]^{-1} \hat{V}\left|\phi_{E}^{m \alpha \pm}\right\rangle\right\rangle,
$$

which can be derived along the same lines as (18); the perturbation operator on the extended Hilbert space is thereby defined as $\langle t|\hat{V}| \psi\rangle\rangle=V_{t}\left|\psi_{t}\right\rangle$. The formal solution of (40) can be found by iteration and reads

$$
\begin{aligned}
\left.\left|\phi_{E}^{m \alpha \pm}\right\rangle\right\rangle & \left.=\sum_{k=0}^{\infty}\left[\left[E_{m}-\hat{H}_{0} \pm i \varepsilon\right]^{-1} \hat{V}\right]^{k}\left|\varphi_{E}^{m \alpha \pm}\right\rangle\right\rangle \\
& \left.=\left[1-\left[E_{m}-\hat{H}_{0} \pm i \varepsilon\right]^{-1} \hat{V}\right]^{-1}\left|\varphi_{E}^{m \alpha \pm}\right\rangle\right\rangle \\
& \left.\left.=\left|\varphi_{E}^{m \alpha \pm}\right\rangle\right\rangle+\left[E_{m}-\hat{H} \pm i \varepsilon\right]^{-1} \hat{V}\left|\varphi_{E}^{m \alpha \pm}\right\rangle\right\rangle .
\end{aligned}
$$

Using the (40) and (41), we can now establish the orthogonality relation for the Floquet scattering vectors,

$$
\begin{aligned}
\left\langle\left\langle\phi_{E^{\prime}}^{n \beta \pm} \mid \phi_{E}^{m \alpha \pm}\right\rangle\right\rangle & =\left\langle\left\langle\varphi_{E^{\prime}}^{n \beta \pm} \mid \phi_{E}^{m \alpha \pm}\right\rangle\right\rangle+\frac{\left\langle\left\langle\varphi_{E^{\prime}}^{n \beta \pm}|\hat{V}| \phi_{E}^{m \alpha \pm}\right\rangle\right\rangle}{E_{n}^{\prime}-E_{m} \mp i \varepsilon} \\
& =\left\langle\left\langle\varphi_{E^{\prime}}^{n \beta \pm} \mid \varphi_{E}^{m \alpha \pm}\right\rangle\right\rangle=\delta_{n m} \delta_{\alpha \beta} \delta_{E-E^{\prime}},
\end{aligned}
$$


and the connecting relations between outgoing and incoming vectors,

$$
\begin{aligned}
& \left\langle\left\langle\phi_{E^{\prime}}^{n \beta \mp} \mid \phi_{E}^{m \alpha \pm}\right\rangle\right\rangle=\left\langle\left\langle\varphi_{E^{\prime}}^{n \beta \mp} \mid \phi_{E}^{m \alpha \pm}\right\rangle\right\rangle \\
& \quad+\frac{\left\langle\left\langle\varphi_{E^{\prime}}^{n \beta \mp}|\hat{V}| \phi_{E}^{m \alpha \pm}\right\rangle\right\rangle}{E_{n}^{\prime}-E_{m} \pm i \varepsilon}=\left\langle\left\langle\varphi_{E^{\prime}}^{n \beta \mp} \mid \varphi_{E}^{m \alpha \pm}\right\rangle\right\rangle \\
& \quad \mp \frac{2 i \varepsilon}{\left(E_{m}-E_{n}^{\prime}\right)^{2}+\varepsilon^{2}}\left\langle\left\langle\varphi_{E^{\prime}}^{n \beta \mp}|\hat{V}| \phi_{E}^{m \alpha \pm}\right\rangle\right\rangle \\
& \quad=\left(\delta_{m n} S_{E}^{\alpha \beta \pm} \mp 2 \pi i\left\langle\left\langle\varphi_{E_{m-n}}^{n \beta \mp}|\hat{V}| \phi_{E}^{m \alpha \pm}\right\rangle\right\rangle\right) \delta_{E_{m}-E_{n}^{\prime}} .
\end{aligned}
$$

Here, we followed the same steps as in the derivations of the (22) and (23). In the (43), $S_{E}^{\alpha \beta \pm}$ denotes the scattering amplitudes for the stationary Hamiltonian $H$.

\subsection{Floquet Scattering Amplitudes I: General Properties}

The Floquet scattering amplitudes are defined as

$$
\left\langle\left\langle\phi_{E^{\prime}}^{0 \beta \mp} \mid \phi_{E}^{m \alpha \pm}\right\rangle\right\rangle \equiv \mathcal{S}_{m, E}^{\alpha \beta \pm} \delta_{E_{m}-E^{\prime}},
$$

where $\mathcal{S}_{m, E}^{\alpha \beta-}=\overline{\mathcal{S}}_{-m, E_{m}}^{\beta \alpha+}$. They satisfy the unitarity conditions

$$
\sum_{n \gamma} \mathcal{S}_{m-n, E_{n}}^{\gamma \beta \mp} \mathcal{S}_{n, E}^{\alpha \gamma \pm}=\delta_{m 0} \delta_{\alpha \beta}
$$

and the symmetry relation

$$
\mathcal{S}_{m, E}^{\alpha \beta \pm}=\tilde{\mathcal{S}}_{-m, E_{m}}^{\beta \alpha \pm}
$$

where the double tilde indicates the reversal of both external magnetic fields and driving protocols. In the following, we will show how these results can be derived within the framework of Floquet scattering theory. Note that, throughout this article, we understand that sums over the mode index run over all integers and that the Floquet scattering amplitudes are zero if their energy argument is not positive.

The unitarity conditions (45) follow from the completeness relation for the Floquet scattering vectors,

$$
\left.\sum_{m \alpha} \mathrm{d} E\left|\phi_{E}^{m \alpha \pm}\right\rangle\right\rangle\left\langle\left\langle\phi_{E}^{m \alpha \pm}\right|=1,\right.
$$

where 1 stands for the identity operator on the extended Hilbert space and the symbolic notation

$$
\sum_{m \alpha} \mathrm{d} E \equiv \sum_{m \alpha} \int_{0}^{\infty} \mathrm{d} E
$$

has been introduced for convenience. We thus have ${ }^{4}$

$$
\begin{aligned}
\left\langle\left\langle\phi_{E^{\prime}}^{0 \beta \pm} \mid \phi_{E}^{m \alpha \pm}\right\rangle\right\rangle & =\delta_{m 0} \delta_{\alpha \beta} \delta_{E_{m}-E^{\prime}} \\
& =\sum_{n \gamma} \mathrm{d}{E^{\prime \prime}}^{\prime}\left\langle\left\langle\phi_{E^{\prime}}^{0 \beta \pm} \mid \phi_{E^{\prime \prime}}^{n \gamma \mp}\right\rangle\right\rangle\left\langle\left\langle\phi_{E^{\prime \prime}}^{n \gamma \mp} \mid \phi_{E}^{m \alpha \pm}\right\rangle\right\rangle \\
& =\left(\sum_{n \gamma} \mathcal{S}_{n, E_{m-n}}^{\gamma \beta \mp} \mathcal{S}_{m-n, E}^{\alpha \gamma \pm}\right) \delta_{E_{m}-E^{\prime}} .
\end{aligned}
$$

and shifting the summation index $n$ yields the result (45).

To derive the symmetry relation (46), we first observe that the free outgoing and incoming Floquet scattering vectors are connected by time reversal, i.e.

$$
\left.\left.\hat{\Theta}\left|\varphi_{E}^{m \alpha \pm}\right\rangle\right\rangle=\left|\tilde{\varphi}_{E}^{m \alpha \mp}\right\rangle\right\rangle,
$$

as can be easily verified with the help of (35) and the definition of the time-reversal operator on the extended Hilbert space, $\langle t|\hat{\Theta}| \psi\rangle\rangle \equiv \Theta\left|\psi_{-t}\right\rangle$. Consequently, acting on the solution of the Floquet-Lippmann-Schwinger equation, (41), with $\hat{\Theta}$ yields ${ }^{5}$

$$
\left.\left.\hat{\Theta}\left|\phi_{E}^{m \alpha \pm}\right\rangle\right\rangle=\left|\tilde{\phi}_{E}^{m \alpha \mp}\right\rangle\right\rangle,
$$

where we have used the identity $\hat{\Theta} \hat{V}=\tilde{V} \hat{\Theta}$ with the time-reversed perturbation operator being defined as $\langle t|\tilde{V}| \psi\rangle\rangle \equiv \tilde{V}_{-t}\left|\psi_{t}\right\rangle$. This result finally implies

$$
\begin{aligned}
\left\langle\left\langle\phi_{E^{\prime}}^{0 \beta \mp} \mid \phi_{E}^{m \alpha \pm}\right\rangle\right\rangle & =\left\langle\left\langle\hat{\Theta} \tilde{\tilde{\phi}}_{E^{\prime}}^{0 \beta \pm} \mid \hat{\Theta} \tilde{\tilde{\phi}}_{E}^{m \alpha \mp}\right\rangle\right\rangle \\
& =\left\langle\left\langle\tilde{\phi}_{E}^{m \alpha \mp} \mid \tilde{\phi}_{E^{\prime}}^{0 \beta \pm}\right\rangle\right\rangle=\tilde{S}_{-m, E_{m}}^{\beta \alpha \pm} \delta_{E_{m}-E^{\prime}}
\end{aligned}
$$

and thus, by comparison with the definition (44), the symmetries (46).

\subsection{Floquet Scattering Amplitudes II: Perturbation Theory}

The framework of our Floquet-Lippmann-Schwinger theory makes it possible to derive a systematic expansion of the Floquet scattering amplitudes in powers of the dynamical potential. To this end, we first compare the definitions (44) with the relations (43) to obtain the explicit expressions

$$
\mathcal{S}_{m, E}^{\alpha \beta \pm}=\delta_{m 0} S_{E}^{\alpha \beta \pm} \mp 2 \pi i\left\langle\left\langle\varphi_{E_{m}}^{0 \beta \mp}|\hat{V}| \phi_{E}^{m \alpha \pm}\right\rangle\right\rangle .
$$

$\overline{4 \text { Note that }}\left\langle\left\langle\phi_{E^{\prime \prime}}^{n \gamma \mp} \mid \phi_{E}^{m \alpha \pm}\right\rangle\right\rangle=\left\langle\left\langle\phi_{E^{\prime \prime}}^{0 \gamma \mp} \mid \phi_{E}^{m-n \alpha \pm}\right\rangle\right\rangle$.

5 Recall that a single tilde indicates the reversal of magnetic fields only and a double tilde includes the reversal of driving protocols. 
Inserting the series representation (41) of the Floquet scattering vector $\left.\left|\phi_{E}^{m \alpha \pm}\right\rangle\right\rangle$ into this formula now yields the expansion

$$
\begin{aligned}
S_{m, E}^{\alpha \beta \pm} & =\delta_{m 0} S_{E}^{\alpha \beta \pm} \\
& \mp 2 \pi i \sum_{l=0}^{\infty}\left\langle\left\langle\varphi_{E_{m}}^{0 \beta \mp}\left|\hat{V}\left[\left[E_{m}-\hat{H}_{0} \pm i \varepsilon\right]^{-1} \hat{V}\right]^{l}\right| \varphi_{E}^{m \alpha \pm}\right\rangle\right\rangle,
\end{aligned}
$$

This result is analogous to the Born series in standard scattering theory [27]. Taking into account only first-order corrections gives the Floquet-Born approximation

$$
\begin{aligned}
S_{m, E}^{\alpha \beta \pm} & \simeq \delta_{m 0} S_{E}^{\alpha \beta \pm} \mp 2 \pi i\left\langle\left\langle\varphi_{E_{m}}^{0 \beta \mp}|\hat{V}| \varphi_{E}^{m \alpha \pm}\right\rangle\right\rangle \\
& =\delta_{m 0} S_{E}^{\alpha \beta \pm} \mp \frac{2 \pi i}{\tau} \int_{0}^{\tau} \mathrm{d} t\left\langle\varphi_{E_{m}}^{\beta \mp}\left|V_{t}\right| \varphi_{E}^{\alpha \pm}\right\rangle u_{t}^{m},
\end{aligned}
$$

which is justified if the amplitude of the external potential variations are small compared to the carrier energy.

\subsection{Scattering Wave Functions}

The physical content of the Floquet scattering states can be understood from their asymptotic wave functions. To derive their structure, we first use the Floquet-LippmannSchwinger (40) and the completeness relation for the free Floquet scattering vectors,

$$
\left.\oint_{m \alpha} \mathrm{d} E\left|\varphi_{E}^{m \alpha \pm}\right\rangle\right\rangle\left\langle\left\langle\varphi_{E}^{m \alpha \pm}\right|=1,\right.
$$

to connect the lead wave functions of the Floquet scattering states with the lead wave functions (5) of the stationary scattering states,

$$
\begin{aligned}
\phi_{E, t}^{\alpha \pm}\left[r_{\beta}\right] \equiv & \left\langle r_{\beta} \mid \phi_{E, t}^{\alpha \pm}\right\rangle=\left\langle\left\langle r_{\beta}, t \mid \phi_{E}^{0 \alpha \pm}\right\rangle\right\rangle \\
= & \left\langle\left\langle r_{\beta}, t \mid \varphi_{E}^{0 \alpha \pm}\right\rangle\right\rangle \\
& +\left\langle\left\langle r_{\beta}, t\left|\left[E-\hat{H}_{0} \pm i \varepsilon\right]^{-1} \hat{V}\right| \phi_{E}^{0 \alpha \pm}\right\rangle\right\rangle \\
= & \varphi_{E}^{\alpha \pm}\left[r_{\beta}\right]+\sum_{m \gamma} \mathrm{d} E^{\prime} \varphi_{E^{\prime}}^{\gamma \mp}\left[r_{\beta}\right] u_{t}^{m} \frac{\left\langle\left\langle\varphi_{E^{\prime}}^{m \gamma \mp}|\hat{V}| \phi_{E}^{0 \alpha \pm}\right\rangle\right\rangle}{E-E_{m}^{\prime} \pm i \varepsilon} .
\end{aligned}
$$

This expression shows that the wave functions $\phi_{E, t}^{\alpha \pm}\left[r_{\beta}\right]$ are invariant under spatial translations by integer multiples of the wave length $\lambda_{E} \equiv 2 \pi / k_{E}$. Therefore, we can evaluate them in the far distance from the scattering region. Plugging (5) into (57) thus yields

$$
\begin{aligned}
\phi_{E, t}^{\alpha \pm}\left[r_{\beta}\right]= & \delta_{\alpha \beta} w_{E}^{\mp}\left[r_{\beta}\right]+S_{E}^{\alpha \beta \pm} w_{E}^{ \pm}\left[r_{\beta}\right] \\
& -\sum_{m \gamma} \mathrm{d} E^{\prime} \frac{\delta_{\beta \gamma} w_{E^{\prime}}^{ \pm}\left[r_{\beta}\right]+S_{E^{\prime}}^{\gamma \beta \mp} w_{E^{\prime}}^{\mp}\left[r_{\beta}\right]}{E^{\prime}-E_{m} \mp i \varepsilon} \\
& \times u_{t}^{-m}\left\langle\left\langle\varphi_{E^{\prime}}^{-m \gamma \mp}|\hat{V}| \phi_{E}^{0 \alpha \pm}\right\rangle\right\rangle \\
\asymp & \delta_{\alpha \beta} w_{E}^{\mp}\left[r_{\beta}\right]+\sum_{m}\left(\delta_{m 0} S_{E}^{\alpha \beta \pm}\right. \\
& \left.\mp 2 \pi i\left\langle\left\langle\varphi_{E_{m}}^{0 \alpha \mp}|\hat{V}| \phi_{E}^{m \alpha \pm}\right\rangle\right\rangle\right) \times w_{E_{m}}^{ \pm}\left[r_{\beta}\right] u_{t}^{-m}
\end{aligned}
$$

Here, we have used Lemma 1c of App. 9 and the symbol $\asymp$ indicates asymptotic equality in the limit $r_{\alpha} \rightarrow \infty$. Finally, inserting the expressions (53) for the Floquet scattering amplitudes gives the wave function

$$
\phi_{E, t}^{\alpha \pm}\left[r_{\beta}\right]=\delta_{\alpha \beta} w_{E}^{\mp}\left[r_{\beta}\right]+\sum_{m} \mathcal{S}_{m, E}^{\alpha \beta \pm} w_{E_{m}}^{ \pm}\left[r_{\beta}\right] u_{t}^{-m} .
$$

This result shows that the outgoing and incoming Floquet scattering states, $\left|\phi_{E, t}^{\alpha+}\right\rangle$ and $\left|\phi_{E, t}^{\alpha-}\right\rangle$, respectively, contain a single incident and escaping wave with wave length $\lambda_{E}$ in the lead $\alpha$. Hence, they represent a carrier with energy $E$ that either enters or leaves the system through the terminal $\alpha$. The Floquet scattering amplitude $S_{m, E}^{\alpha \beta+}$ thus corresponds to the probability amplitude for a transitions from the terminal $\alpha$ to the terminal $\beta$ under the absorption $(m>0)$ or emission $(m<0)$ of $m$ units of energy $\hbar \omega$. Analogously, $S_{m, E}^{\alpha \beta-}$ corresponds to the probability amplitude for that an escaping carrier with energy $E$ in the terminal $\alpha$ was injected into the terminal $\beta$ with an energy surplus $(m>0)$ or deficit $(m<0)$ of $m$ quanta $\hbar \omega$. In this picture, the unitarity condition (45) ensures the conservation of probability currents. The symmetry relation (46) implies that forward and backward processes occur with the same probability amplitude provided that no magnetic field is applied to the system and the driving protocols are invariant under time reversal [15, 24].

We stress that the lead wave functions (59) have not been used to define the Floquet scattering states in our approach; in fact, their structure results from the continuity condition $\lim _{V_{t} \rightarrow 0}\left|\phi_{E, t}^{\alpha \pm}\right\rangle=\left|\varphi_{E}^{\alpha \pm}\right\rangle$, which has been built into the Floquet-Lippmann-Schwinger equation (40). In the same way, the quantization of the energy flux between carriers and driving fields arises naturally from the periodicity condition $\left|\phi_{E, t}^{\alpha \pm}\right\rangle=\left|\phi_{E, t+\tau}^{\alpha \pm}\right\rangle$, which is imposed by the Floquet theorem and encoded in structure of the extended Hilbert space.

Note that the lead wave functions (59) can be used as boundary conditions to determine the incoming and outgoing solutions of the Floquet-Schrödinger equation 
(28) in position representation. For sufficiently simple dynamical potentials, the Floquet scattering amplitudes can thus be found by calculating the Floquet wave functions inside the scattering region and solving a spatiotemporal boundary value problem [39-41].

\section{Matter and Energy Currents}

\subsection{Current Operators}

On the single-particle level, the matter and energy currents that flow at the position $r_{\alpha}$ of the lead $\alpha$ into a multi-terminal conductor are represented by the operators $[42,43]$

$$
\begin{aligned}
& j_{\alpha}^{\rho} \equiv-\frac{1}{2 M}\left\{P, \delta\left[R-r_{\alpha}\right]\right\} \quad \text { and } \\
& j_{\alpha}^{\varepsilon} \equiv-\frac{1}{8 M}\left\{P^{2},\left\{P, \delta\left[R-r_{\alpha}\right]\right\}\right\} .
\end{aligned}
$$

Here, $R$ and $P$ are the position and momentum operators, $M$ denotes the carrier mass and curly brackets indicate the usual anti-commutator. Note that, for convenience, we notationally suppress the dependence of the current operators on the coordinate $r_{\alpha}$ throughout.

As the transport carriers are indistinguishable, the many-body quantum state of a mesoscopic conductor must be either symmetric or antisymmetric under the exchange of two arbitrary carriers. An elegant method to take this constraint into account is provided by the language of second quantization, which can be adopted to our present setup as follows. We first introduce the scattering field operators $\Phi_{E, t}^{\alpha}$ and $\Phi_{E, t}^{\alpha \dagger}$, which annihilate and create a carrier in the outgoing Floquet scattering state $\left|\phi_{E, t}^{\alpha+}\right\rangle$, respectively. For any fixed time $t$, these operators obey the commutation relations

$$
\begin{gathered}
\left\{\Phi_{E^{\prime}, t}^{\beta}, \Phi_{E, t}^{\alpha}\right\}=\left\{\Phi_{E^{\prime}, t}^{\beta^{\dagger}}, \Phi_{E, t}^{\alpha \dagger}\right\}=0 \quad \text { and } \\
\left\{\Phi_{E^{\prime}, t}^{\beta^{\dagger}}, \Phi_{E, t}^{\alpha}\right\}=\left\{\Phi_{E^{\prime}, t}^{\beta}, \Phi_{E, t}^{\alpha \dagger}\right\}=\delta_{\alpha \beta} \delta_{E-E^{\prime}},
\end{gathered}
$$

where we focus on Fermions for the sake of concreteness; the theory for Bosonic carriers can be developed analogously. The many-particle current operators can now be expresses as

$$
\mathrm{J}_{\alpha}^{x}=\underbrace{}_{\beta} \mathrm{d} E \oint_{\gamma} \mathrm{d} E^{\prime} j_{E E^{\prime}, t}^{\chi \alpha, \beta \gamma} \Phi_{E, t}^{\beta \dagger} \Phi_{E^{\prime}, t}^{\gamma},
$$

where $x \equiv \rho, \varepsilon$ and

$$
\begin{aligned}
j_{E E^{\prime}, t}^{\rho \alpha, \beta \gamma} \equiv & \left\langle\phi_{E, t}^{\beta+}\left|j_{\alpha}^{\rho}\right| \phi_{E^{\prime}, t}^{\gamma+}\right\rangle \\
= & \left.\frac{i \hbar}{2 m}\left(\bar{\phi}_{E}^{\beta} \phi_{E^{\prime} ; 1}^{\gamma}-\phi_{E^{\prime}}^{\gamma} \bar{\phi}_{E ; 1}^{\beta}\right)\right|_{r=r_{\alpha}} \\
j_{E E^{\prime}, t}^{\varepsilon \alpha, \beta \gamma} \equiv & \left\langle\phi_{E, t}^{\beta+}\left|j_{\alpha}^{\varepsilon}\right| \phi_{E^{\prime}, t}^{\gamma}\right\rangle \\
= & \frac{i \hbar^{3}}{8 m^{2}}\left(\phi_{E^{\prime}}^{\gamma} \bar{\phi}_{E ; 3}^{\beta}-\phi_{E^{\prime} ; 1}^{\gamma} \bar{\phi}_{E ; 2}^{\beta}\right. \\
& \left.+\bar{\phi}_{E ; 1}^{\beta} \phi_{E^{\prime} ; 2}^{\gamma}-\bar{\phi}_{E}^{\beta} \phi_{E^{\prime} ; 3}^{\gamma}\right)\left.\right|_{r=r_{\alpha}}
\end{aligned}
$$

with $\phi_{E}^{\alpha} \equiv \phi_{E, t}^{\alpha+}[r]$ and $\phi_{E ; l}^{\alpha} \equiv \partial_{r}^{l} \phi_{E, t}^{\alpha+}[r]$. These matrix elements are $\tau$-periodic functions of $t$ and can thus be expanded in a Fourier series,

$$
j_{E E^{\prime}, t}^{\chi \alpha, \beta \gamma} \equiv \sum_{m} k_{E E^{\prime}, m}^{\chi \alpha, \beta \gamma} \exp [i m \omega t],
$$

where the coefficients $k_{E E^{\prime}, m}^{\chi \alpha, \beta \gamma}$ can be determined from the Floquet scattering wave functions (59). Rather than spelling out the corresponding expressions in full generality, we here provide only a specific set of Fourier components that will be needed in the following sections and can be written in the compact form

$$
\begin{aligned}
& k_{E E_{m}, m}^{\chi \alpha, \beta \gamma}=\frac{1}{h}\left(\delta_{\alpha \beta} \delta_{\alpha \gamma} \delta_{m 0} \zeta_{E}^{X}-\sum_{n} \mathcal{S}_{n-m, E_{m}}^{\gamma \alpha+} \overline{\mathcal{S}}_{n, E}^{\beta \alpha+} \zeta_{E_{n}}^{\chi}\right) \\
& \text { with } h \equiv 2 \pi \hbar, \quad \zeta_{E}^{\rho} \equiv 1, \quad \zeta_{E}^{\varepsilon} \equiv E .
\end{aligned}
$$

\subsection{Mean Currents}

We are now ready to calculate the average steady-state currents of matter and energy in a periodically driven multiterminal conductor. To this end, we recall the general formula (1a) for the mean currents,

$$
J_{\alpha}^{x} \equiv \lim _{t \rightarrow \infty} \frac{1}{t} \int_{0}^{t} \mathrm{~d} t^{\prime}\left\langle\mathrm{J}_{\alpha, t^{\prime}}^{x}\right\rangle .
$$

The Heisenberg-picture operator $J_{\alpha, t}^{x}$ thereby describes the flow of particles $(x=\rho)$ or energy $(x=\varepsilon)$ at a given time $t$ and at a given position $r_{\alpha}$ in the lead $\alpha$; angular brackets denote the ensemble average over all possible quantum states of the system.

The formula (66) can be evaluated in two steps. First, transforming the current operators (62) into the Heisenberg picture yields

$$
\begin{aligned}
\mathrm{J}_{\alpha, t}^{X} & \equiv \mathrm{U}_{t}^{\dagger} \mathrm{J}_{\alpha}^{x} \mathrm{U}_{t} \\
& =\oint_{\beta} \mathrm{d} E \sum_{\gamma} \mathrm{d} E^{\prime} j_{E E^{\prime}, t}^{x \alpha, \beta \gamma} \Phi_{E}^{\beta \dagger} \Phi_{E^{\prime}}^{\gamma} \exp \left[i\left(E-E^{\prime}\right) t / \hbar\right],
\end{aligned}
$$


where the unitary operator $\mathrm{U}_{t}$ generates the evolution of the many-particle system from the time 0 to the time $t$. The second line in (67) follows from the time evolution laws for the field operators,

$$
\begin{aligned}
\mathrm{U}_{t}^{\dagger} \Phi_{E, t}^{\alpha} \mathrm{U}_{t} & =\Phi_{E}^{\alpha} \exp [-i E t / \hbar] \text { and } \\
\mathrm{U}_{t}^{\dagger} \Phi_{E, t}^{\alpha \dagger} \mathrm{U}_{t} & =\Phi_{E}^{\alpha} \exp [i E t / \hbar]
\end{aligned}
$$

which, in turn, are a consequence of the fact that the outgoing scattering states $\left|\phi_{E, t}^{\alpha+}\right\rangle$ are solutions of the Floquet Schrödinger equation (28) and thus fulfill ${ }^{6}$

$$
U_{t}\left|\phi_{E}^{\alpha+}\right\rangle=\exp [-i E t / \hbar]\left|\phi_{E, t}^{\alpha+}\right\rangle .
$$

Here, $U_{t}$ is the single-particle time evolution operator. Note that the time argument 0 is omitted throughout for simplicity.

Second, to evaluate the ensemble average in (66), we recall that the outgoing Floquet scattering states $\left|\phi_{E, t}^{\alpha+}\right\rangle$ are populated with non-interacting carriers by a thermochemical reservoir with temperature $T_{\alpha}$ and chemical potential $\mu_{\alpha}$. Hence, provided that all reservoirs are mutually independent, the quantum-statistical average of an ordered pair of one creation and one anihilation operator is given by the grand canonical rule

$$
\begin{aligned}
& \left\langle\Phi_{E}^{\alpha \dagger} \Phi_{E^{\prime}}^{\beta}\right\rangle=\delta_{\alpha \beta} \delta_{E-E^{\prime}} f_{E}^{\alpha}, \\
& \text { where } f_{E}^{\alpha} \equiv \frac{1}{1+\exp \left[\left(E-\mu_{\alpha}\right) / T_{\alpha}\right]}
\end{aligned}
$$

denotes the Fermi function of the reservoir $\alpha$ and Boltzmann's constant is set to 1 throughout; averages of products that contain different numbers of creation and annihilation are zero [8, 9, 24].

Inserting (67) into the formula (66) and using (70) yields

$$
\begin{aligned}
& J_{\alpha}^{x}=\lim _{t \rightarrow \infty} \frac{1}{t} \int_{0}^{t} d t^{\prime} \sum_{\beta} \mathrm{d} E j_{E E, t}^{x \alpha, \beta \beta} f_{E}^{\beta} \\
& =\sum_{\beta} \mathrm{d} E k_{E E, 0}^{\chi \alpha, \beta \beta} f_{E}^{\beta} \text {, }
\end{aligned}
$$

where we have used the Fourier expansion (64) for the second identity. Upon recalling the matrix elements (65),

6 To verify the time evolution laws for the scattering field operators, construct a basis of the many-particle Fock space from the incoming Floquet scattering states $\left|\phi_{E}^{\alpha+}\right\rangle$ and evaluate the corresponding matrix elements of both sides of the (68a) and (68b) with the help of the relation (69). the mean currents can now be expressed in terms of the Floquet scattering amplitudes of the conductor and the Fermi functions of the attached reservoirs,

$$
J_{\alpha}^{X}=\frac{1}{h} \oint_{\beta} \mathrm{d} E\left(\delta_{\alpha \beta} \zeta_{E}^{X}-\sum_{m}\left|\mathcal{S}_{m, E}^{\beta \alpha+}\right|^{2} \zeta_{E_{m}}^{X}\right) f_{E}^{\beta} .
$$

This formula, which holds arbitrary far from equilibrium, shows that the conductance properties of a coherent multi-terminal system are fully determined by its Floquet scattering amplitudes. In the limit $V_{t} \rightarrow 0$, where the Floquet scattering amplitudes become equal to the stationary ones according to (53), it reduces to the standard Landauer-Büttiker formula.

The physical consistency of the current formula (72) derives from the sum rules

$$
\sum_{m \alpha}\left|\mathcal{S}_{-m, E_{m}}^{\alpha \beta+}\right|^{2}=1 \quad \text { and } \quad \sum_{m \alpha}\left|\mathcal{S}_{m, E}^{\beta \alpha+}\right|^{2}=1,
$$

which follow directly from the unitarity conditions for the Floquet scattering amplitudes, (45). By using the first of these relations, (72) can be rewritten in the form

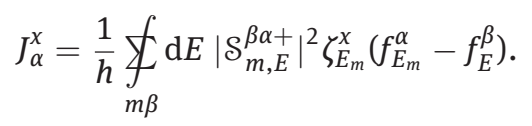

This result shows that the mean currents indeed vanish in equilibrium, i.e. if all reservoirs are at the same temperature and chemical potential and the external driving fields are turned off. Furthermore, by summing both sides of (72) over the terminal index and using the second sum rule in (73), we recover the fundamental conservation laws for matter and energy,

$$
\sum_{\alpha} J_{\alpha}^{\rho}=0 \text { and } \quad \sum_{\alpha} J_{\alpha}^{\varepsilon}=-\Pi_{\mathrm{ac}} .
$$

The average power that is injected into the system through the external driving, $\Pi_{a c}$, thereby admits the microscopic expression

$$
\Pi_{\mathrm{ac}} \equiv \frac{1}{\tau} \oint_{m \alpha \beta} \mathrm{d} E\left|\mathcal{S}_{m, E}^{\beta \alpha+}\right|^{2} m f_{E}^{\beta} .
$$

\subsection{Zero-Frequency Noise}

The zero-frequency noise, or noise power, of the matter and energy currents in a multi-terminal conductor is given by the general formula

$$
\begin{aligned}
P_{\alpha \beta}^{x y} & \equiv \lim _{t \rightarrow \infty} \frac{1}{t} \int_{0}^{t} \mathrm{~d} t^{\prime} \int_{0}^{t} \mathrm{~d} t^{\prime \prime}\left\langle\left(\mathrm{J}_{\alpha, t^{\prime}}^{x}-J_{\alpha}^{x}\right)\left(\mathrm{J}_{\beta, t^{\prime \prime}}^{y}-J_{\beta}^{y}\right)\right\rangle \\
& =\lim _{t \rightarrow \infty} \frac{1}{t} \int_{0}^{t} \mathrm{~d} t^{\prime} \int_{0}^{t} \mathrm{~d} t^{\prime \prime}\left\langle\left\langle\mathrm{J}_{\alpha, t^{\prime}}^{x} ; \mathrm{J}_{\beta, t^{\prime \prime}}^{y}\right\rangle\right\rangle
\end{aligned}
$$


for $x=\rho, \varepsilon$ and $y=\rho, \varepsilon$. Here, the notation

$$
\langle\langle A ; B\rangle\rangle \equiv\langle A B\rangle-\langle A\rangle\langle B\rangle
$$

has been introduced for the correlation function of the observables $A$ and $B$. The quantity $P_{\alpha \beta}^{x y}$ can be calculated with the same techniques as the mean currents. In the first step, we use (67) to express the time-dependent current operators in terms of the scattering field operators and obtain

$$
\begin{aligned}
& P_{\alpha \beta}^{x y}=\lim _{t \rightarrow \infty} \frac{1}{t} \int_{0}^{t} d t^{\prime} \int_{0}^{t} d t^{\prime \prime} \oint_{\gamma_{1}} \mathrm{~d} E_{1} \cdots \sum_{\gamma_{4}} \mathrm{~d} E_{4} \\
& j_{E_{1} E_{2}, t^{\prime}}^{x \alpha, \gamma_{1} \gamma_{2}} \cdot j_{E_{3} E_{4}, t^{\prime \prime}}^{y \beta, \gamma_{3} \gamma_{4}}\left\langle\left\langle\Phi_{E_{1}}^{\gamma_{1}{ }^{\dagger}} \Phi_{E_{2}}^{\gamma_{2}} ; \Phi_{E_{3}}^{\gamma_{3}{ }^{\dagger}} \Phi_{E_{4}}^{\gamma_{4}}\right\rangle\right\rangle \\
& \times \exp \left[i\left(E_{1}-E_{2}\right) t^{\prime} / \hbar\right] \exp \left[i\left(E_{3}-E_{4}\right) t^{\prime \prime} / \hbar\right]
\end{aligned}
$$

with dots being inserted to improve readability. The correlation function of the scattering field operators in (79) can be evaluated using the finite-temperature version of Wick's theorem [44], which implies

$$
\begin{aligned}
& \left\langle\left\langle\Phi_{E_{1}}^{\gamma_{1} \dagger} \Phi_{E_{2}}^{\gamma_{2}} ; \Phi_{E_{3}}^{\gamma_{3} \dagger} \Phi_{E_{4}}^{\gamma_{4}}\right\rangle\right\rangle=\left\langle\Phi_{E_{1}}^{\gamma_{1} \dagger} \Phi_{E_{4}}^{\gamma_{4}}\right\rangle\left\langle\Phi_{E_{2}}^{\gamma_{2}} \Phi_{E_{3}}^{\gamma_{3} \dagger}\right\rangle \\
& =\delta_{\gamma_{1} \gamma_{4}} \delta_{E_{1}-E_{4}} f_{E_{1}}^{\gamma} \cdot \delta_{\gamma_{2} \gamma_{3}} \delta_{E_{2}-E_{3}}\left(1-f_{E_{2}}^{\delta}\right) .
\end{aligned}
$$

Here, we have used the commutation rules (61) and the grand canonical averaging rule (70) for the last identity. After inserting (80) and the Fourier expansion of the current matrix elements (64) into (79), we can carry out the time integrals. This step yields

$$
\begin{aligned}
P_{\alpha \beta}^{x y} & =\lim _{t \rightarrow \infty} \oint_{m \gamma} \mathrm{d} E \sum_{n \delta} \mathrm{d} E^{\prime} k_{E E^{\prime}, m}^{\chi \alpha, \gamma} \cdot k_{E^{\prime} E,-n}^{y \beta, \delta \gamma} \cdot f_{E}^{\gamma}\left(1-f_{E^{\prime}}^{\delta}\right) \\
& \times t \cdot \frac{\exp \left[-i\left(E^{\prime}-E_{m}\right) t / \hbar\right]-1}{\left(E^{\prime}-E_{m}\right) t / \hbar} \frac{\exp \left[i\left(E^{\prime}-E_{n}\right) t / \hbar\right]-1}{\left(E^{\prime}-E_{n}\right) t / \hbar} .
\end{aligned}
$$

Upon taking the limit $t \rightarrow \infty$ with the help of Lemma 2 of App. 9, this expression simplifies to the compact result

$$
P_{\alpha \beta}^{x y}=h \sum_{m \gamma \delta} \mathrm{d} E k_{E E_{m}, m}^{\chi \alpha, \gamma \delta} \cdot \bar{k}_{E E_{m}, m}^{y \beta, \gamma \delta} \cdot f_{E}^{\gamma}\left(1-f_{E_{m}}^{\delta}\right),
$$

where we have applied the relation $k_{E_{m} E,-m}^{y \beta, \delta \gamma}=\bar{k}_{E E_{m}, m}^{y \beta,}$.

The zero-frequency noise can now be expressed in terms of the Floquet scattering amplitudes of the driven conductor and the Fermi functions of the reservoirs. To this end, we insert the matrix elements (65) into (82). After some algebra, we thus obtain the explicit formula

$$
\begin{aligned}
P_{\alpha \beta}^{x y} & =\frac{1}{h} \sum_{m} \mathrm{~d} E\left(\delta_{m 0} \delta_{\alpha \beta} \zeta_{E}^{x} \zeta_{E}^{y} f_{E}^{\prime \alpha}\right. \\
& -\left|\mathcal{S}_{m, E}^{\alpha \beta+}\right|^{2} \zeta_{E}^{x} \zeta_{E_{m}}^{y} f_{E}^{\prime \alpha}-\left|\mathcal{S}_{m, E}^{\beta \alpha+}\right|^{2} \zeta_{E_{m}}^{x} \zeta_{E}^{y} f_{E}^{\prime \beta} \\
& \left.+\sum_{\gamma \delta} \mathcal{W}_{m, E}^{x \alpha, \gamma \delta} \overline{\mathcal{W}}_{m, E}^{y \beta, \gamma \delta} f_{E}^{\gamma}\left(1-f_{E_{m}}^{\delta}\right)\right),
\end{aligned}
$$

where we have introduced the abbreviations

$$
{f^{\prime}}_{E}^{\alpha} \equiv f_{E}^{\alpha}\left(1-f_{E}^{\alpha}\right), \quad \mathcal{W}_{m, E}^{\alpha \alpha, \gamma \delta} \equiv \sum_{n} \overline{\mathcal{S}}_{n, E}^{\gamma \alpha+} \mathcal{S}_{n-m, E_{m}}^{\delta \alpha+} \zeta_{\xi_{n}}^{X}
$$

for convenience. ${ }^{7}$

In order to analyze the physical content of the key result (83), it is instructive to divide the noise power into two contributions, $P_{\alpha \beta}^{x y} \equiv Q_{\alpha \beta}^{x y}+N_{\alpha \beta}^{x y}$, that are given by $Q_{\alpha \beta}^{x y} \equiv R_{\alpha \beta}^{x y}+R_{\alpha \beta}^{y x}$ and $N_{\alpha \beta}^{x y} \equiv W_{\alpha \beta}^{x y}+C_{\alpha \beta}^{x y}$ with $^{8}$

$$
\begin{aligned}
& R_{\alpha \beta}^{x y} \equiv \frac{1}{h} \oint_{m} \mathrm{~d} E\left(\delta_{m 0} \delta_{\alpha \beta} \zeta_{E}^{X}-\left|\mathcal{S}_{m, E}^{\beta \alpha+}\right|^{2} \zeta_{E_{m}}^{X}\right) \zeta_{E}^{y} f_{E}^{\prime \beta}, \\
& W_{\alpha \beta}^{x y} \equiv \frac{1}{2 h} \oint_{m \gamma \delta} \mathrm{d} E \mathcal{W}_{m, E}^{x \alpha, \gamma \delta} \overline{\mathcal{W}}_{m, E}^{y \beta, \gamma \delta}\left(f_{E}^{\gamma}-f_{E_{m}}^{\delta}\right)^{2}, \\
& C_{\alpha \beta}^{x y} \equiv \delta_{\alpha \beta} \frac{1}{h} \sum_{m \gamma} \mathrm{d} E\left|\mathcal{S}_{m, E}^{\gamma \alpha+}\right|^{2} \zeta_{E_{m}}^{\chi} \zeta_{E_{m}}^{y}\left(f_{E}^{\prime \gamma}-{f^{\prime}}_{E_{m}}^{\alpha}\right) .
\end{aligned}
$$

Here, the thermal noise, or Nyquist-Johnson noise, $Q_{\alpha \beta}^{x y}$, results from thermal fluctuations in the incoming beams of carriers that emerge from the reservoirs. It remains finite in equilibrium but vanishes at zero temperature, where thermal fluctuations are frozen out and ${f^{\prime}}_{E}^{\alpha}=$ 09 . By contrast, the non-equilibrium noise $N_{\alpha \beta}^{x y}$ vanishes if no external driving is applied to the conductor and all reservoirs have the same temperature and chemical potential. Its first component, the shot noise $W_{\alpha \beta}^{x y}$, which persists in the zero-temperature limit, describes fluctuations in the matter and energy currents due to the probabilistic nature of carrier transmissions and photon exchange between carriers and driving fields in the quantum regime. Finally, the non-equilibrium correction, $C_{\alpha \beta}^{x y}$, which vanishes at zero temperature, accounts for modulations of the thermal fluctuations in the outgoing beams of carriers due to thermochemical biases and periodic driving.

7 The formula (83) shows that the noise power $P_{\alpha \beta}^{x y}$ is real and obeys the symmetry $P_{\alpha \beta}^{x y}=P_{\beta \alpha}^{y x}$. These properties cannot be a priori expected as the current correlation function in (77) is, in general, not symmetric with respect to the current operators. In fact, the antisymmetric, imaginary part of this correlation function is wiped out only when the limit $t \rightarrow \infty$ is taken in (81). The finite-frequency noise must therefore be derived from symmeterized correlation functions, for details see [9, 22, 24].

8 To prove that the quantities $Q_{\alpha \beta}^{x y}$ and $N_{\alpha \beta}^{x y}$ indeed sum up to the total noise power (83), use the sum rules (73), the unitarity conditions (45) and shift the integration variables as needed.

9 To be precise, we have $f_{E}^{\prime \alpha} \rightarrow 0$ for $E \neq \mu_{\alpha}$ and $f_{E}^{\prime \alpha} \rightarrow 1 / 4$ for $E=$ $\mu_{\alpha}$ in the limit $T_{\alpha} \rightarrow 0$. Note that $f_{E}^{\prime \alpha}$ is the negative derivative of the Fermi function $f_{E}^{\alpha}$ with respect to $\left(E-\mu_{a}\right) / T_{\alpha}$. 
As a final remark for this section, we note that, although we have focused here on matter and energy currents, our analysis applies to any set of generalized currents that can be represented by operators of the form

$$
\mathrm{J}_{\alpha}^{\prime x}=\sum_{y \beta} c_{\alpha \beta}^{x y} \mathrm{~J}_{\beta}^{y}
$$

with real coefficients $c_{\alpha \beta}^{x y}$. Specifically, the corresponding mean currents and the zero-frequency noise can be obtained directly from the formulas (72) and (83) through the transformation rules

$$
\begin{aligned}
J_{\alpha}^{\prime x} & \equiv \lim _{t \rightarrow \infty} \frac{1}{t} \int_{0}^{t} \mathrm{~d} t^{\prime}\left\langle\mathrm{J}_{\alpha, t}^{\prime x}\right\rangle=\sum_{y \beta} c_{\alpha \beta}^{x y} J_{\beta}^{y}, \\
P_{\alpha \beta}^{\prime x y} & \equiv \lim _{t \rightarrow \infty} \frac{1}{t} \int_{0}^{t} \mathrm{~d} t^{\prime} \int_{0}^{t} \mathrm{~d} t^{\prime \prime}\left\langle\left\langle\mathrm{J}_{\alpha, t^{\prime}}^{\prime x} ; \mathrm{J}_{\beta, t^{\prime \prime}}^{\prime y}\right\rangle\right\rangle \\
& =\sum_{u \gamma} \sum_{v \delta} c_{\alpha \gamma}^{x u} c_{\beta \delta}^{y v} P_{\gamma \delta}^{u v},
\end{aligned}
$$

where $u=\rho, \varepsilon, v=\rho, \varepsilon$. The thermal and quantum components of the transformed noise power, $P_{\alpha \beta}^{\prime x y} \equiv Q_{\alpha \beta}^{\prime x y}+$ ${N^{\prime}}_{\alpha \beta}^{\prime x y} \equiv R_{\alpha \beta}^{\prime x y}+R_{\beta \alpha}^{\prime y x}+{W^{\prime}}_{\alpha \beta}^{\prime x y}+{C^{\prime}}_{\alpha \beta}^{x y}$, can thus be identified by analogy as

$$
A_{\alpha \beta}^{\prime x y}=\sum_{u \gamma} \sum_{v \delta} c_{\alpha \gamma}^{x u} c_{\beta \delta}^{y v} A_{\gamma \delta}^{u v}
$$

for $A=Q, N, R, W, C$.

\section{Thermodynamics}

\subsection{The First Law}

The first law for periodically driven multi-terminal conductors follows directly from the conservation laws (75) and can be formulated as

$$
\sum_{\alpha} J_{\alpha}^{q}+\Pi_{\mathrm{ac}}-\Pi_{\mathrm{el}}=0
$$

with $J_{\alpha}^{q} \equiv J_{\alpha}^{\varepsilon}-\mu_{\alpha} J_{\alpha}^{\rho} \quad$ and $\quad \Pi_{\mathrm{e} l} \equiv \sum_{\alpha}\left(\mu-\mu_{\alpha}\right) J_{\alpha}^{\rho}$,

where $\mu$ denotes the base level of the chemical potential. It governs the balance between the thermal energy that is injected into the system by the reservoirs through the heat currents $J_{\alpha}^{q}$, the mechanical power provided by the time dependent driving fields, $\Pi_{\mathrm{a} c}$, and the electrical power generated through the redistribution of carriers between the reservoirs, $\Pi_{\mathrm{el}}$. Within the Floquet scattering approach, the first law (89) is an immediate consequence of the sum rules (73).

\subsection{The Second Law}

The second law requires that the average rate of entropy production that is caused by the transport process is nonnegative, that is [45]

$$
\sigma \equiv-\sum_{\alpha} J_{\alpha}^{q} / T_{\alpha} \geq 0
$$

A simple demonstration that the Floquet scattering approach is consistent with this constraint uses only the sum rules (73) and the fact that the Fermi distribution is the derivative of a convex function, for details see [46, 47]. In the following, we provide an alternative proof, which also shows that the dissipation rate $\sigma$ can only become zero if all currents in the system vanish.

Our proof is inspired by methods that are usually employed to derive bounds on quantum entropy functions, for details see [48]. The key idea is to express the rate of entropy production in terms of the binary entropy function

$$
\eta[a] \equiv-a \ln [a]-(1-a) \ln [1-a]
$$

and its first derivative, where $0 \leq a \leq 1$. A quadratic lower bound on $\sigma$ can then be obtained from a simple argument involving Taylor's theorem. We proceed as follows. First, we use the formula (72) for the mean currents and the sum rules (73) to rewrite $\sigma$ as

$$
\begin{aligned}
h \sigma= & \sum_{m \alpha \beta} \mathrm{d} E\left|S_{m, E}^{\beta \alpha+}\right|^{2}\left(\left(E_{m}-\mu_{\alpha}\right) / T_{\alpha}-\left(E-\mu_{\beta}\right) / T_{\beta}\right) f_{E}^{\beta} \\
= & \sum_{m \alpha \beta} \mathrm{d} E\left|S_{m, E}^{\beta \alpha+}\right|^{2}\left(\left(\ln \left[f_{E}^{\beta}\right]-\ln \left[f_{E_{m}}^{\alpha}\right]\right) f_{E}^{\beta}\right. \\
& \left.+\left(\ln \left[1-f_{E}^{\beta}\right]-\ln \left[1-f_{E_{m}}^{\alpha}\right]\right)\left(1-f_{E}^{\beta}\right)\right) \\
= & \oint_{m \alpha \beta} \mathrm{d} E\left|S_{m, E}^{\beta \alpha+}\right|^{2}\left(\eta\left[f_{E_{m}}^{\alpha}\right]-\eta\left[f_{E}^{\beta}\right]+\eta_{1}\left[f_{E_{m}}^{\alpha}\right]\left(f_{E}^{\beta}-f_{E_{m}}^{\alpha}\right)\right)
\end{aligned}
$$

with $\eta_{l}[a] \equiv \partial_{a}^{l} \eta[a]$. By Taylor's theorem, there now exists a $g$ between $f_{E}^{\beta}$ and $f_{E_{m}}^{\alpha}$ such that

$$
\begin{aligned}
& \eta\left[f_{E_{m}}^{\alpha}\right]-\eta\left[f_{E}^{\beta}\right]+\eta_{1}\left[f_{E_{m}}^{\alpha}\right]\left(f_{E}^{\beta}-f_{E_{m}}^{\alpha}\right) \\
& =-\eta_{2}[g]\left(f_{E}^{\beta}-f_{E_{m}}^{\alpha}\right)^{2} / 2 .
\end{aligned}
$$

Since the Fermi function takes only values between 0 and 1 , the number $g$ must also lie in this interval. Hence, we have $-\eta_{2}[g]=1 / g+1 /(1-g) \geq 4$ and therefore

$$
\sigma \geq \frac{2}{h} \sum_{m \alpha \beta} \mathrm{d} E\left|\mathcal{S}_{m, E}^{\beta \alpha+}\right|^{2}\left(f_{E_{m}}^{\alpha}-f_{E}^{\beta}\right)^{2}
$$

upon combining the (92) and (93). 
The bound (94) shows that, first, the rate of entropy production can indeed not become negative within the Floquet scattering approach and, second, that $\sigma$ is zero if and only if the integrand in (94) vanishes for all energies $E$ and all combinations of the indices $m, \alpha, \beta$. Under this condition, however, all energy and particle currents must also be zero according to (74). We stress that this result, which was obtained here without any assumptions on the behavior of the system under time reversal, should, though intuitively expectable, not be regarded as trivial. In fact, the question whether or not dissipationless currents can exist in normal conducting mesoscopic systems with broken time reversal symmetry has been the subject of an active debate in recent years [11].

Finally, we note that the rate or entropy production (90) is in fact the mean value of a generalized current that is represented by the operator

$$
\mathrm{J}_{\sigma} \equiv-\sum_{\alpha}\left(\mathrm{J}_{\alpha}^{\varepsilon}-\mu_{\alpha} \mathrm{J}_{\alpha}^{\rho}\right) / T_{\alpha} .
$$

Therefore, the formalism developed in Section 6.3 can be applied to investigate whether not only the average but also the fluctuations, or even higher-oder cumulants, of the entropy production are subject to universal bounds. This problem has recently been studied for stationary mesoscopic conductors [49, 50]. We leave it to future research to extend this approach to periodically driven systems.

\subsection{Green-Kubo Relations}

The Green-Kubo relations are a cornerstone result of nonequilibrium statistical mechanics. They make it possible to express the linear response coefficients that quantify the variations of mean currents due to a small changes of the thermodynamic forces that drive the system away from equilibrium in terms of integrated equilibrium correlation functions of the involved currents $[2,45]$. As our final topic in this article, we will now show how this fundamental relationship arises naturally within the framework of Floquet scattering theory.

The thermodynamic forces, or affinities, for a transport process are defined as gradients in the thermodynamic variables that form entropy-conjugate pairs with the conserved quantities of the system. For a multi-terminal conductor, these objects can be identified with the thermochemical biases between the external reservoirs,

$$
F_{\alpha}^{\rho} \equiv \mu_{\alpha} / T_{\alpha}-\mu / T \quad \text { and } \quad F_{\alpha}^{\varepsilon} \equiv 1 / T-1 / T_{\alpha},
$$

where, $\mu$ and $T$ denote the base chemical potential and temperature. Using these definitions, the rate of entropy production (90) can be divided into a mechanical part, $\sigma_{\mathrm{ac}} \equiv \Pi_{\mathrm{ac}} / T$, and a thermal one, $\sigma_{\mathrm{th}} \equiv \sigma-\sigma_{\mathrm{ac}}$, which now assumes the characteristic bilinear form of irreversible thermodynamics [45],

$$
\sigma_{\mathrm{th}}=\sum_{\alpha} F_{\alpha}^{\rho} J_{\alpha}^{\rho}+F_{\alpha}^{\varepsilon} J_{\alpha}^{\varepsilon} .
$$

Several proposals were made to extend this structure to the total rate of entropy production by associating the mechanical perturbation with an effective current and a generalized affinity, which, depending on the scheme, corresponds to the mean applied work [51] or either the amplitude [52-54] or the frequency $[47,55]$ of the periodic driving fields. For the purpose of our analysis, however, it is sufficient to focus on the conventional thermal currents and affinities appearing in (97).

To establish the Green-Kubo relations for multiterminal systems we first calculate the response coefficients

$$
L_{\alpha \beta}^{x y}=\partial_{\beta}^{y} J_{\alpha}^{X}=\frac{1}{h} \oiint_{m} \mathrm{~d} E\left(\delta_{m 0} \delta_{\alpha \beta} \zeta_{E}^{X}-\left|\mathcal{S}_{m, E}^{\beta \alpha+}\right|^{2} \zeta_{E_{m}}^{X}\right) \zeta_{E}^{y} f_{E}^{\prime \beta},
$$

where we have used the current formula (72) and the symbol $\partial_{\beta}^{y}$ indicates the derivative with respect to the affinity $F_{\beta}^{y}$. Upon comparing this expression with the components of the current noise given in the (85), we find that $L_{\alpha \beta}^{x y}=R_{\alpha \beta}^{x y}$ and thus

$$
L_{\alpha \beta}^{x y}+L_{\beta \alpha}^{y x}=Q_{\alpha \beta}^{x y}=P_{\alpha \beta}^{x y}-N_{\alpha \beta}^{x y} .
$$

Hence, the symmetric part of the response coefficients (98) is identical to the thermal noise, even if the transport process takes place far from equilibrium. In equilibrium, i.e. for $F_{\alpha}^{x}=0$ and $V_{t}=0$, the non-equilibrium noise $N_{\alpha \beta}^{x y}$ vanishes and the relation (99) becomes $\left.\left(L_{\alpha \beta}^{x y}+L_{\beta \alpha}^{y x}\right)\right|_{\text {eq }}=$ $\left.P_{\alpha \beta}^{x y}\right|_{\text {eq. }}$. Moreover, provided that no magnetic fields are applied to the sample, we recover the Onsager symmetry $\left.L_{\alpha \beta}^{x y}\right|_{\text {eq }}=\left.L_{\beta \alpha}^{y x}\right|_{\text {eq }}$, as can be easily verified from the property (14) of the stationary scattering amplitudes [45]. We thus arrive at the standard form of the Green-Kubo relations for multi-terminal conductors,

$$
\left.2 L_{\alpha \beta}^{x y}\right|_{\text {eq }}=\left.\lim _{t \rightarrow \infty} \frac{1}{t} \int_{0}^{t} \mathrm{~d} t^{\prime} \int_{0}^{t} \mathrm{~d} t^{\prime \prime}\left\langle\left\langle J_{\alpha, t^{\prime}}^{x} ; \mathrm{J}_{\beta, t^{\prime \prime}}^{y}\right\rangle\right\rangle\right|_{\text {eq }} .
$$

In order to extend the result (100) to non-equilibrium situations and systems with broken time-reversal symmetry, we have to express the coefficient $L_{\alpha \beta}^{x y}$ as an integrated 
correlation function that involves the current operator $\mathbf{J}_{\alpha}^{X}$. That is, we look for an observable $\mathbf{I}_{\alpha}^{X}$ that fulfills

$$
L_{\alpha \beta}^{x y}=\lim _{t \rightarrow \infty} \frac{1}{t} \int_{0}^{t} \mathrm{~d} t^{\prime} \int_{0}^{t} \mathrm{~d} t^{\prime \prime}\left\langle\left\langle\mathrm{J}_{\alpha, t^{\prime}}^{x} ; \mathrm{I}_{\beta, t^{\prime \prime}}^{y}\right\rangle\right\rangle .
$$

A minimal choice for such a variable is given by

$$
\mathrm{I}_{\alpha}^{\chi} \equiv \int_{0}^{\infty} \mathrm{d} E \int_{0}^{\infty} \mathrm{d} E^{\prime} i_{E E^{\prime}}^{\chi \alpha} \Phi_{E, t}^{\alpha \dagger} \Phi_{E^{\prime}, t}^{\alpha},
$$

where the energy dependent weights,

$$
\begin{array}{r}
i_{E E^{\prime}}^{\rho \alpha} \equiv \frac{1}{2 h}\left[k_{E} k_{E^{\prime}}\right]^{-\frac{1}{2}}\left(k_{E}+k_{E^{\prime}}\right), \\
i_{E E^{\prime}}^{\varepsilon \alpha} \frac{1}{4 h}\left[k_{E} k_{E^{\prime}}\right]^{-\frac{1}{2}}\left(k_{E}+k_{E^{\prime}}\right)\left(E+E^{\prime}\right),
\end{array}
$$

are found by replacing the scattering wave functions $\phi_{E, t}^{\alpha+}[r]$ in (63a) and (63b) with the plane waves $w_{E}^{-}[r]$; recall (6) for the definition of $w_{E}^{-}[r]$ and $k_{E}$. This operator can be easily shown to satisfy the condition (101) by following the lines of Section 6.3. It describes the gross influx of matter $(x=\rho)$ or energy $(x=\varepsilon)$ from the reservoir $\alpha$ and thus provides a physically transparent nonequilibrium generalization of the Green-Kubo relation (100), which covers even systems with broken time reversal symmetry. From a practical perspective, the result (101) makes it possible to infer the time-integrated correlation function between net currents and gross influx, which are otherwise hard to access, by measuring the variations of mean currents in response to small changes of the thermochemical biases (96).

We conclude this section by pointing out that the bilinear decomposition (97) of $\sigma_{\text {th }}$ into affinities and currents is not unique. In fact, for any set of generalized currents and affinities,

$$
\begin{aligned}
& J_{\alpha}^{\prime x}=\sum_{y \beta}=c_{\alpha \beta}^{x y} J_{\beta}^{y} \text { and } F_{\alpha}^{\prime x}=\sum_{y \beta} \mathrm{d}_{\alpha \beta}^{x y} F_{\beta}^{y} \\
& \text { with } \sum_{u \gamma} c_{\gamma \alpha}^{u x} \mathrm{~d}_{\gamma \beta}^{u y}=\delta_{x y} \delta_{\alpha \beta},
\end{aligned}
$$

the thermal rate of entropy production assumes the standard form $\sigma_{\mathrm{th}}=\sum_{x \alpha} J_{\alpha}^{\prime \prime} F_{\alpha}^{\prime x}$. In particular, for the specific choice $c_{\alpha \beta}^{\rho x}=\delta_{\alpha \beta} \delta_{x \rho}$ and $c_{\alpha \beta}^{\varepsilon x}=\delta_{\alpha \beta}\left(\delta_{x \varepsilon}-\mu_{\alpha} \delta_{x \rho}\right)$, the energy currents are replaced by the heat current; that is, we have $J_{\alpha}^{\prime \varepsilon}=J_{\alpha}^{\varepsilon}-\mu_{\alpha} J_{\alpha}^{\rho}=J_{\alpha}^{q}$ and ${F^{\prime}}_{\alpha}^{\varepsilon}=\left(\mu_{\alpha}-\mu\right) / T \equiv$ $F_{\alpha}^{q}$. The Green-Kubo relations (100) and their generalized counterparts (101) are invariant under such linear transformations provided that the generalized influx operators are identified as $\mathrm{I}_{\alpha}^{\prime x}=\sum_{y \beta} c_{\alpha \beta}^{x y} \mathrm{I}_{\alpha}^{x}$. This result follows from the fact that the response coefficients (98) obey the same transformation rules as the zero-frequency current noise and its components, which are given in the (87b) and (88). Specifically, we have

$$
L_{\alpha \beta}^{\prime x y} \equiv \partial_{\beta}^{\prime y} J_{\alpha}^{\prime x}=\sum_{u \gamma} \sum_{v \delta} c_{\alpha \gamma}^{x u} c_{\beta \delta}^{y u} L_{\gamma \delta}^{u v}
$$

as can be easily verified by inspection.

\section{Perspectives and Challenges}

\subsection{Adiabatic Perturbation Theory}

In Section 5.4, we have shown how the Floquet scattering amplitudes can be calculated order by order in the dynamical potential. This approach is well justified if the periodic variations of the scattering potential are small compared to the typical carrier energies. For practical purposes, however, an adiabatic perturbation scheme, where the frequency rather than the amplitude of the driving fields plays the role of the expansion parameter, is often more suitable.

Such a theory can be developed as follows. Consider an approaching or escaping carrier with energy $E$ in the terminal $\alpha$. If the dynamical potential is practically constant during the dwell time of this carrier inside the sample, its transition through the system at the time $t$ is described by the frozen scattering states $\left|\kappa_{E, t}^{\alpha \pm}\right\rangle[56,57]$. These states are solutions of the stationary Schrödinger equation

$$
H_{t}\left|\kappa_{E, t}^{\alpha \pm}\right\rangle=E\left|\kappa_{E, t}^{\alpha \pm}\right\rangle
$$

and satisfy the boundary conditions

$$
\left\langle r_{\beta} \mid \kappa_{E}^{\alpha \pm}\right\rangle \equiv \kappa_{E}^{\alpha \pm}\left[r_{\beta}\right]=\delta_{\alpha \beta} w_{E}^{\mp}\left[r_{\beta}\right]+S_{E, t}^{\alpha \beta \pm} w_{E}^{ \pm}\left[r_{\beta}\right]
$$

with the frozen scattering amplitudes given by

$$
\left\langle\kappa_{E^{\prime}, t}^{\beta \mp} \mid \kappa_{E, t}^{\alpha \pm}\right\rangle=S_{E, t}^{\alpha \beta \pm} \delta_{E-E^{\prime}} .
$$

The corresponding quasi-static Floquet scattering amplitudes are the Fourier components of these objects, i.e.

$$
\mathcal{S}_{0 m, E}^{\alpha \beta \pm}=\frac{1}{\tau} \int_{0}^{\tau} \mathrm{d} t S_{E, t}^{\alpha \beta \pm} u_{t}^{m} .
$$

This result follows by comparing (107) with (59) and assuming that the carrier energy is practically constant during the transition through the sample.

The expression (109) can be interpreted as the zeroth order of an expansion of the Floquet scattering amplitudes 
in the photon energy $\hbar \omega$. The first-order term of this series can be determined from a phenomenological ansatz of the form [16]

$$
\mathcal{S}_{1 m, E}^{\alpha \beta \pm}=\frac{\hbar \omega}{\tau} \int_{0}^{\tau} \mathrm{d} t m \partial_{E} S_{E, t}^{\alpha \beta \pm} u_{t}^{m} \pm \hbar \omega \mathcal{A}_{m, E}^{\alpha \beta \pm} .
$$

Here, the first term accounts for small changes in the carrier energy during the transition and the correction $\mathcal{A}_{m, E}^{\alpha \beta \pm}$ is chosen such that the approximated Floquet scattering amplitudes obey the unitarity conditions (45).

This scheme proved quite effective for various practical applications [15, 24]. How it can be derived from a systematic perturbation theory, which would make it possible to also calculate higher-order terms, however, is not immediately clear. As a first attempt, we might try to adapt the Lippmann-Schwinger formalism of Section 5.2 by mimicking the adiabatic perturbation theory for systems with discrete spectrum $[34,58,59]$. To this end, the free scattering vectors (35) have to be replaced with their frozen counterparts,

$$
\left.\left\langle t \mid \kappa_{E}^{m \alpha \pm}\right\rangle\right\rangle \equiv u_{t}^{m}\left|\kappa_{E_{m}, t}^{\alpha \pm}\right\rangle .
$$

The roles of the free effective Hamiltonian and the perturbation are then assumed by the operators $\hat{K}$ and $\hat{D}$, respectively, which are defined as $\langle t|\hat{K}| \psi\rangle\rangle \equiv H_{t}\left|\psi_{t}\right\rangle$ and $\langle t|\hat{D}| \psi\rangle\rangle \equiv-i \hbar \partial_{t}\left|\psi_{t}\right\rangle$. Upon repeating the derivations of Sec. 5.2, we thus find that the Floquet scattering amplitudes, up to second-order contributions in $\hat{D}$, read

$$
\begin{aligned}
\mathcal{S}_{m, E}^{\alpha \beta \pm} & =\frac{1}{\tau} \int_{0}^{\tau} \mathrm{d} t S_{E_{m}, t}^{\alpha \beta \pm} u_{t}^{m} \mp 2 \pi i\left\langle\left\langle\kappa_{E_{m}}^{0 \beta \mp}|\hat{D}| \kappa_{E}^{m \alpha \pm}\right\rangle\right\rangle \\
& =\frac{1}{\tau} \int_{0}^{\tau} \mathrm{d} t S_{E_{m}, t}^{\alpha \beta \pm} u_{t}^{m} \pm \hbar \omega \int_{0}^{\tau} \mathrm{d} t\left\langle\dot{\kappa}_{E_{m}, t}^{\beta \mp} \mid \kappa_{E_{m}, t}^{\alpha \pm}\right\rangle u_{t}^{m} \\
& =\frac{1}{\tau} \int_{0}^{\tau} \mathrm{d} t S_{E, t}^{\alpha \beta \pm} u_{t}^{m}+\frac{\hbar \omega}{\tau} \int_{0}^{\tau} \mathrm{d} t m \partial_{E} S_{E, t}^{\alpha \beta \pm} u_{t}^{m} \\
& \pm \hbar \omega \int_{0}^{\tau} \mathrm{d} t\left\langle\dot{\kappa}_{E, t}^{\beta \pm} \mid \kappa_{E, t}^{\alpha \pm}\right\rangle u_{t}^{m}+\mathcal{O}\left[(\hbar \omega)^{2}\right] .
\end{aligned}
$$

Hence, we indeed recover the zeroth- and fist-order terms (109) and (110). However, this result must be taken with a grain of salt, as the correction term in (112), which involves the time derivative of the frozen scattering state $\left|\kappa_{E, t}^{\beta \pm}\right\rangle$, is generally divergent. Therefore, the expression (112) should not be regarded as a proper expansion of the Floquet scattering amplitudes.
The singular behavior of the last term in (112) arises because the time derivative $\hat{D}$, in contrast to the dynamical potential $\hat{V}$, which vanishes outside the scattering region, constitutes an unbounded operator on $\hat{\mathcal{H}}$. To overcome this problem, it might be necessary to invoke techniques of singular perturbation theory, adiabatic gauge potentials $[60,61]$ or a transformation of the scattering amplitudes into the time domain; the latter approach lead to a consistent first-order expansion in [62]. We leave it as a challenge for future studies to derive a systematic adiabatic perturbation theory by further developing the formalism presented in this article.

\subsection{Thermal Machines}

The Floquet scattering formalism provides a general platform to explore the performance of thermal nano-devices. As a concrete example, we might consider a quantum heat engine that consists of a driven sample and two reservoirs with equal chemical potential $\mu$ and different temperatures $T_{1} \equiv T_{\mathrm{c}}$ and $T_{2} \equiv T_{\mathrm{h}}>T_{\mathrm{c}}$. Here, we imagine that the variations of the scattering potential are caused by the motion of mechanical degrees of freedom like a mesoscopic paddle wheel, which perform work against some external load [63-65]. The thermodynamic performance of such a machine is determined by two benchmark parameters, its mean power output $-\Pi_{a c}$ and its efficiency $\eta \equiv$ $-\Pi_{\mathrm{a} c} / J_{\mathrm{h}}^{q}$. The latter figure is thereby subject the Carnot bound

$$
\eta \leq \eta_{\mathrm{C}} \equiv 1-T_{\mathrm{c}} / T_{\mathrm{h}}
$$

which follows from the second law, $\sigma=J_{\mathrm{h}}^{q} F_{\mathrm{h}}^{q}+\Pi_{\mathrm{ac}} / T_{\mathrm{c}} \geq$ 0 , and can be attained only in the quasi-static limit, where $-\Pi_{a c}$ goes to zero ${ }^{10}$.

From a practical perspective, it is therefore important to determine the maximum efficiency, at which a nanoengine can deliver a given power output. For autonomous, i.e. thermoelectric, heat engines such bounds have been found by seeking constraints on the total rate of entropy production that go beyond the second law [66-70], or by explicitly optimizing the scattering amplitudes of the sample [71-75]. The first strategy has also been applied in studies of piston-type heat engines, which use a closed working system, and lead to the general trade-off relation

$$
\eta\left(\eta_{\mathrm{C}}-\eta\right) \geq-\Pi / \Theta
$$

10 This result follows from the fact that the rate of entropy production $\sigma$ vanishes only if all currents are zero, see Section 7.2. 
between efficiency $\eta$ and power output $-\Pi$; here, $\Theta>0$ is a system-specific constant $[52,53,68,69]$. First steps towards an extension of this bound to paddle-wheel type quantum engines, which are driven by a continuous flow of carriers, have been made under the assumptions of slowly varying driving fields and small thermochemical biases $[47,55]$. A universal and physically transparent performance bound that covers also devices operating far from equilibrium is, however, still lacking.

\subsection{Thermodynamic Uncertainty Relations}

Thermodynamic uncertainty relations describe a tradeoff between dissipation and precision in non-equilibrium processes. Specifically, for a time-homogeneous Markov process that obeys detailed balance, the inequality

$$
\sigma \varepsilon^{2} \geq 2 \quad \text { with } \quad \varepsilon \equiv \sqrt{P / J^{2}}
$$

holds for arbitrary currents with mean value $J$ and fluctuations, or noise power, $P$, where $\sigma$ denotes the total rate of entropy production and $\varepsilon$ the relative uncertainty of the current $J[76,77]$. This bound, which was first discovered for biomolecular processes, does, however, not apply to periodically driven systems, systems with broken timereversal symmetry or in the quantum regime [78-84]. In order to close these gaps, a whole variety of generalized thermodynamic uncertainty relations have been proposed over the last years, see for instance [25, 85-93].

A particularly transparent result was recently obtained in [89], where the frequency dependent bound

$$
\sigma_{\omega} \varepsilon_{\omega}^{2} \geq 2\left[1-\omega\left(\partial_{\omega} J_{\omega}\right) / J_{\omega}\right]^{2}
$$

was derived for periodically driven Markov jump processes. Whether or not this result can be extended to coherent mesoscopic conductors, or whether the relation (115) can be generalized for such systems by other means are compelling questions, which can be systematically investigated within the theoretical framework presented in this article. Further research in this direction promises valuable insights on how quantum effects can be exploited to control the thermodynamic cost of precision in transport processes. However, this endeavor can be expected to be challenging, as general properties of the Floquet scattering amplitudes that go beyond the ones discussed in Section 5 are hard to establish and specific models for which they can be determined exactly are scarce.

Acknowledgements: The author acknowledges insightful discussions with E. Potanina, M. Moskalets, K. Saito, and
U. Seifert. The research leading to the results presented in this article has received funding from the Academy of Finland (Contract No. 296073), the Japan Society for the Promotion of Science through a Postdoctoral Fellowship for Research in Japan (Fellowship ID: P19026), the University of Nottingham, Nottingham Research Fellowship and from UK Research and Innovation through a Future Leaders Fellowship (Grant Reference: MR/S034714/1).

\section{A Appendix: Some Helpful Lemmas}

Lemma 1a: Let $F_{z}^{ \pm}$be a complex function that is bounded and holomorphic on the stripe $D^{ \pm} \equiv[0, \infty) \times[0, \pm i R]$ with $R>0$. Then, for any $v>0$, we have

$$
\begin{aligned}
& \lim _{\varepsilon \rightarrow 0} \int_{0}^{\infty} d u \frac{\exp [ \pm i x u]}{u^{2}-v^{2} \mp i \varepsilon} F_{u}^{ \pm} \asymp \frac{\pi i \exp [ \pm i x v]}{v} F_{v}^{ \pm}, \\
& \lim _{\varepsilon \rightarrow 0} \int_{0}^{\infty} d u \frac{\exp [ \pm i x u]}{u^{2}+v^{2} \mp i \varepsilon} F_{u}^{ \pm} \asymp 0
\end{aligned}
$$

in the limit $x \rightarrow \infty$.

Proof. We proceed in two steps. First, we close the integration path in the complex plane as shown in Figure 3 and observe that

$$
\begin{aligned}
& \int_{\Gamma_{r}^{ \pm}} \mathrm{d} z \frac{\exp [ \pm i x z]}{z^{2}-v^{2} \mp i \varepsilon} F_{z}^{ \pm} \asymp \int_{0}^{\infty} \mathrm{d} u \frac{\exp [ \pm i x u]}{u^{2}-v^{2} \mp i \varepsilon} F_{u}^{ \pm}, \\
& \int_{\Gamma_{r}^{ \pm}} \mathrm{d} z \frac{\exp [ \pm i x z]}{z^{2}+v^{2} \mp i \varepsilon} F_{z}^{ \pm} \asymp \int_{0}^{\infty} \mathrm{d} u \frac{\exp [ \pm i x u]}{u^{2}+v^{2} \mp i \varepsilon} F_{u}^{ \pm}
\end{aligned}
$$

for $x \rightarrow \infty$, since the integrand on the left-hand side is exponentially suppressed in $x$ on either the upper $(+)$ or
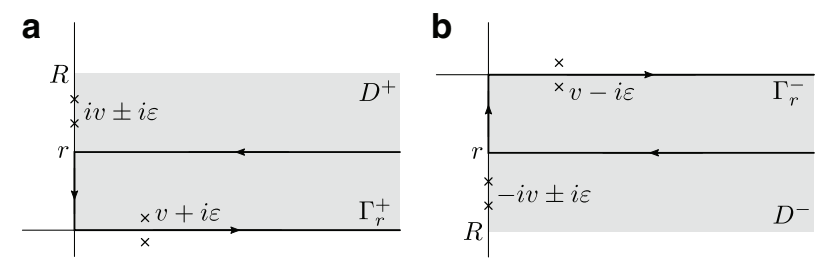

Figure 3: Graphical illustration of the integration contours used in the proof of Lemma 1. (a) The contour $\Gamma_{r}^{+}$encircles a rectangle with height $r$ and infinite width, whose lower edge falls on the positive real axis. Crosses indicate the singularities of the integrands in the (119a) and (119b). (b) The same picture for the contour $\Gamma_{r}^{-}$. 
the lower (-) half plane. Second, using Cauchy's theorem to evaluate the contour integral yields

$$
\begin{aligned}
& \lim _{\varepsilon \rightarrow 0} \int_{\Gamma_{R}^{ \pm}} \mathrm{d} z \frac{\exp [ \pm i x z]}{z^{2}-v^{2} \mp i \varepsilon} F_{z}^{ \pm} \\
& =\lim _{\varepsilon \rightarrow 0} \int_{\Gamma_{R}^{ \pm}} \mathrm{d} z\left[\frac{1}{z-v \mp i \varepsilon}-\frac{1}{z+v \pm i \varepsilon}\right] \frac{\exp [ \pm i x z]}{2 v} F_{z}^{ \pm} \\
& = \pm \frac{\pi i \exp [ \pm i x v]}{v} F_{v}^{ \pm} \text {and } \\
& \lim _{\varepsilon \rightarrow 0} \int_{\Gamma_{r}^{ \pm}} \mathrm{d} z \frac{\exp [ \pm i x z]}{z^{2}+v^{2} \mp i \varepsilon} F_{z}^{ \pm} \\
& =\lim _{\varepsilon \rightarrow 0} \int \mathrm{d} z\left[\frac{1}{z-i v \mp i \varepsilon}-\frac{1}{z+i v \pm i \varepsilon}\right] \\
& \quad \frac{\exp [ \pm i x z]}{2 v} F_{z}^{ \pm}=0
\end{aligned}
$$

where we set $r=R$ in (119a) and $0<r<v$ in (119b).

Lemma 1b: For $F_{z}^{ \pm}$as in Lemma $1 a$ and $w \neq 0$ being real, we have

$$
\lim _{\varepsilon \rightarrow 0} \int_{0}^{\infty} d u \frac{\exp [\mp i x u]}{u^{2}+w \mp i \varepsilon} F_{u}^{ \pm} \asymp 0
$$

in the limit $x \rightarrow \infty$.

Proof. Set $w=-v^{2}$ for $w<0$ and $w=v^{2}$ for $w>0$ and repeat the steps of the proof of Lemma 1 .

Lemma 1c: For $F_{z}^{ \pm}$as in Lemma 1a, $G_{z}^{ \pm} \equiv F_{\sqrt{z}}^{ \pm}$and $w \neq 0$ being real, we have

$$
\begin{gathered}
\lim _{\varepsilon \rightarrow 0} \int_{0}^{\infty} d u \frac{\exp [ \pm i x \sqrt{u}]}{u-w \mp i \varepsilon} \frac{G_{u}^{ \pm}}{\sqrt{u}} \\
\asymp \pm 2 \pi i \frac{\exp [ \pm i x \sqrt{w}] G_{w}^{ \pm}}{\sqrt{w}}, \\
\lim _{\varepsilon \rightarrow 0} \int_{0}^{\infty} d u \frac{\exp [\mp i x \sqrt{u}]}{u-w \mp i \varepsilon} \frac{G_{u}^{ \pm}}{\sqrt{u}} \asymp 0
\end{gathered}
$$

in the limit $x \rightarrow \infty$.

Proof. Change the integration variable to $s \equiv \sqrt{u}$ and apply the Lemmas $1 \mathrm{a}$ and $1 \mathrm{~b}$.

Lemma 2: Let $F_{u}$ be a test function on the real axis and define $\theta_{u} \equiv(1-\exp [-i u]) /(i u)$. Then, for any integers $m$ and $n$, we have

$$
\lim _{x \rightarrow \infty} \int d u x \cdot \theta_{(u-m) x} \bar{\theta}_{(u-n) x} F_{u}=2 \pi \delta_{m n} F_{m}
$$

Proof. We first rewrite the left-hand side of (122) as

$$
\begin{aligned}
& \lim _{x \rightarrow \infty} \int \mathrm{d} u x \cdot\left|\theta_{(u-m) x}\right|^{2} \frac{\bar{\theta}_{(u-n) x}}{\bar{\theta}_{(u-m) x}} F_{u} \\
& =\lim _{x \rightarrow \infty} \int \mathrm{d} u x \cdot \operatorname{sinc}^{2}[(u-m) x / 2] \frac{\bar{\theta}_{(u-n) x}}{\bar{\theta}_{(u-m) x}} F_{u},
\end{aligned}
$$

where $\operatorname{sinc}[u] \equiv \sin [u] / u$. Next, we observe that the function $\operatorname{sinc}^{2}[u]$ assumes only non-negative values and obeys

$$
\begin{array}{r}
\lim _{x \rightarrow \infty} \int_{|u| \leq \varepsilon} \mathrm{d} u x \cdot \operatorname{sinc}^{2}[u x]=\pi \text { and } \\
\lim _{x \rightarrow \infty} x \cdot \operatorname{sinc}^{2}[u x]=0 \text { for } \varepsilon<|u|<1 / \varepsilon,
\end{array}
$$

where $\varepsilon>0$. Consequently, we have [36]

$$
\begin{aligned}
& \lim _{x \rightarrow \infty} \int \mathrm{d} u x \cdot \sin c^{2}[(u-m) x / 2] \frac{\bar{\theta}_{(u-n) x}}{\bar{\theta}_{(u-m) x}} F_{u} \\
& =2 \pi \lim _{x \rightarrow \infty} \bar{\theta}_{(m-n) x} F_{m}=2 \pi \delta_{m n} F_{m},
\end{aligned}
$$

where we used that $\lim _{u \rightarrow 0} \theta_{u}=1$ and $\lim _{x \rightarrow \infty} \theta_{m x}=0$ for any $m \neq 0$.

\section{References}

[1] R. Kubo, Rep. Prog. Phys. 29, 255 (1966).

[2] R. Kubo, M. Toda, and N. Hashitsume, Statistical Physics II Nonequilibrium Statistical Mechanics, 2nd ed., Springer, Tokyo 1998.

[3] U. M. B. Marconi, A. Puglisi, L. Rondoni, and A. Vulpiani, Phys. Rep. 461, 111 (2008).

[4] U. Seifert, Phys. Rev. Lett. 104, 138101 (2010).

[5] G. B. Lesovik and I. A. Sadovskyy, Phys.-Usp. 54, 1007 (2014).

[6] P. A. Mello and N. Kumar, Quantum Transport in Mesoscopic Systems, 1st ed., Oxford University Press, Oxford 2004.

[7] Y. V. Nazarov and Y. M. Blanter, Quantum Transport - Introduction to Nanoscience, 1st ed., Cambridge University Press, Cambridge 2009.

[8] Y. M. Blanter and M. Büttiker, Phys. Rep. 336, 1 (2000).

[9] M. Büttiker, Phys. Rev. B 46, 12485 (1992).

[10] M. Büttiker, Y. Imry, R. Landauer, and S. Pinhas, Phys. Rev. B 31, 6207 (1985).

[11] G. Benenti, G. Casati, K. Saito, and R. S. Whitney, Phys. Rep. 694, 1 (2017).

[12] P. Gaspard, New J. Phys. 15, 115014 (2013).

[13] P. Gaspard, New J. Phys. 17, 045001 (2015).

[14] P. Gaspard, Ann. Phys. (Berlin) 527, 663 (2015).

[15] M. Moskalets and M. Büttiker, Phys. Rev. B 66, 205320 (2002).

[16] M. V. Moskalets, Scattering Matrix Approach to Quantum Transport, 1st ed., Imperial College Press, London 2012.

[17] M. H. Pedersen and M. Buttiker, Phys. Rev. B 58, 12993 (1998).

[18] M. Wagner, Phys. Rev. Lett. 85, 174 (2000).

[19] M. Wagner and F. Sols, Phys. Rev. Lett. 83, 4377 (1999). 
[20] M. F. Ludovico, L. Arrachea, M. Moskalets, and D. Sánchez, Entropy 18, 419 (2016).

[21] S. Vinjanampathy and J. Anders, Contemp. Phys. 57, 545 (2016).

[22] M. Moskalets, Phys. Rev. Lett. 112, 206801 (2014).

[23] M. Moskalets and M. Büttiker, Phys. Rev. B 66, 035306 (2002).

[24] M. Moskalets and M. Büttiker, Phys. Rev. B 70, 245305 (2004).

[25] J. M. Horowitz and T. R. Gingrich, Nat. Phys. 16, 15 (2020).

[26] L. E. Ballentine, Quantum Mechanics: A Modern Development, 1st ed., World Scientific, Singapore 1998.

[27] R. G. Newton, Scattering Theory of Waves and Particles, 2nd ed., Springer Science+Business Media, New York 1982.

[28] H. Sambe, Phys. Rev. A 7, 2203 (1973).

[29] J. S. Howland, Indiana U. Math. J. 28, 471 (1979).

[30] N. Moiseyev and R. Lefebvre, Phys. Rev. A 58, 4218 (1998).

[31] U. Peskin and N. Moiseyev, Phys. Rev. A 49, 3712 (1994).

[32] H. U. Baranger and A. D. Stone, Phys. Rev. B 40, 8169 (1989).

[33] M. Mintchev, L. Santoni, and P. Sorba, Ann. Phys. (Berlin) 529, 1600274 (2017).

[34] L. I. Schiff, Quantum Mechanics, 3rd ed., McGraw-Hill Book Company, New York 1968.

[35] G. F. Mazenko, Nonequilibrium Statistical Mechanics, 1st ed., Wiley-VCH Verlag GmbH \& Co KGaA, Weinheim, Weinheim 2006.

[36] W. Appel, Mathematics for Physics and Physicists, 1st ed., Princeton University Press, Princeton, NJ 2007.

[37] J. H. Shirley, Phys. Rev. 138, B979 (1965).

[38] Y. B. Zel'dovich, Sov. Phys. JETP 24, 1006 (1967).

[39] W. Li and L. E. Reichel, Phys. Rev. B 60, 15732 (1999).

[40] D. F. Martinez and L. E. Reichl, Phys. Rev. B 64, 245315 (2001).

[41] M. Wagner, Phys. Rev. B 49, 16544 (1994).

[42] R. J. Hardy, Phys. Rev. 132, 168 (1963).

[43] A. Kugler, Z. Phys. 198, 236 (1967).

[44] G. Giulianni and G. Vignale, Quantum Theory of the Electron Liquid, Cambridge University Press, Cambridge 2005.

[45] H. B. Callen, Thermodynamics and an Introduction to Thermostatics, 2nd ed., John Wiley \& Sons, New York 1985.

[46] G. Nenciu, J. Math. Phys. 48, 033302 (2007).

[47] E. Potanina, M. Moskalets, C. Flindt, and K. Brandner, preprint arXiv:1906.04297 (2019).

[48] M. Ohya and D. Petz, Quantum Entropy and Its Use, 1st ed., Springer-Verlang, Berlin, Heidelberg 1993.

[49] M. Mintchev, L. Santoni, and P. Sorba, Phys. Rev. E 96, 052124 (2017).

[50] M. Mintchev, L. Santoni, and P. Sorba, Ann. Phys. (Berlin) 530, 1800170 (2018).

[51] Y. Izumida and K. Okuda, Eur. Phys. J. B 77, 499 (2010).

[52] K. Brandner, K. Saito, and U. Seifert, Phys. Rev. X 5, 031019 (2015).

[53] K. Brandner and U. Seifert, Phys. Rev. E 93, 062134 (2016).

[54] K. Proesmans and C. van den Broeck, Phys. Rev. Lett. 115, 090601 (2015).

[55] M. F. Ludovico, F. Battista, F. von Oppen, and L. Arrachea, Phys. Rev. B 93, 075136 (2016).

[56] V. Gasparian, T. Christen, and M. Büttiker, Phys. Rev. A 54, 4022 (1996).

[57] C. Texier, Physica E 82, 16 (2016).
[58] V. Cavina, A. Mari, and V. Giovannetti, Phys. Rev. Lett. 119, 050601 (2017).

[59] M. S. Sarandy and D. A. Lidar, Phys. Rev. A 71, 012331 (2005).

[60] M. Kolodrubetz, D. Sels, P. Mehta, and A. Polkovnikov, Phys. Rep. 697, 1 (2017).

[61] P. Weinberg, M. Bukov, L. D’Alessio, A. Polkovnikov, S. Vajna, and M. Kolodrubetz, Phys. Rep. 688, 1 (2017).

[62] M. Thomas, T. Karzig, S. V. Kusminskiy, G. Zaránd, and F. von Oppen, Phys. Rev. B 86, 195419 (2012).

[63] L. Arrachea and F. von Oppen, Physica E 82, 247 (2016).

[64] A. Bruch, S. V. Kusminskiy, G. Refael, and F. von Oppen, Phys. Rev. B 97, 195411 (2018).

[65] R. Bustos-Marún, G. Refael, and F. von Oppen, Phys. Rev. Lett. 111, 060802 (2013).

[66] K. Brandner and U. Seifert, Phys. Rev. E 91, 012121 (2015).

[67] P. Pietzonka and U. Seifert, Phys. Rev. Lett. 120, 190602 (2018).

[68] N. Shiraishi and K. Saito, J. Stat. Phys. 174, 433 (2019).

[69] N. Shiraishi, K. Saito, and H. Tasaki, Phys. Rev. Lett. 117, 190601 (2016).

[70] R. S. Whitney, Phys. Rev. B 87, 115404 (2013).

[71] P. P. Hofer and B. Sothmann, Phys. Rev. B 91, 195406 (2015).

[72] P. Samuelsson, S. Kheradsoud, and B. Sothmann, Phys. Rev. Lett. 118, 256801 (2017).

[73] R. Sánchez, B. Sothmann, and A. N. Jordan, Phys. Rev. Lett. 114, 146801 (2015).

[74] R. S. Whitney, Phys. Rev. Lett. 112, 130601 (2014).

[75] R. S. Whitney, Phys. Rev. B 91, 115425 (2015).

[76] A. C. Barato and U. Seifert, Phys. Rev. Lett. 114, 158101 (2015).

[77] T. R. Gingrich, J. M. Horowitz, N. Perunov, and J. L. England, Phys. Rev. Lett. 116, 120601 (2016).

[78] A. C. Barato and U. Seifert, Phys. Rev. X 6, 041053 (2016).

[79] K. Brandner, T. Hanazato, and K. Saito, Phys. Rev. Lett. 120, 090601 (2018).

[80] V. Holubec and A. Ryabov, Phys. Rev. Lett. 121, 120601 (2018).

[81] K. Ptaszyński, Phys. Rev. B 98, 085425 (2018).

[82] B. K. Agarwalla and D. Segal, Phys. Rev. B 98, 155438 (2018).

[83] S. Saryal, H. M. Friedman, D. Segal, and B. K. Agarwalla, Phys. Rev. E 100, 042101 (2019).

[84] J. Liu and D. Segal, Phys. Rev. E 99, 062141 (2019).

[85] A. C. Barato, R. Chetrite, A. Faggionato, and D. Gabrielli, New J. Phys. 20, 103023 (2018).

[86] F. Carollo, R. L. Jack, and J. P. Garrahan, Phys. Rev. Lett. 122, 130605 (2019).

[87] G. Guarnieri, G. T. Landi, S. R. Clark, and J. Goold, Phys. Rev. Research 1, 033021 (2019).

[88] Y. Hasegawa and T. Van Vu, Phys. Rev. Lett. 123, 110602 (2019).

[89] T. Koyuk and U. Seifert, Phys. Rev. Lett. 122, 230601 (2019).

[90] T. Koyuk, U. Seifert, and P. Pietzonka, J. Phys. A: Math. Theor. 52, 02 LTO2 (2019).

[91] K. Macieszczak, K. Brandner, and J. P. Garrahan, Phys. Rev. Lett. 121, 130601 (2018).

[92] K. Proesmans and C. Van den Broeck, Europhys. Lett. 119, 20001 (2017).

[93] A. M. Timpanaro, G. Guarnieri, J. Goold, and G. T. Landi, Phys. Rev. Lett. 123, 090604 (2019). 